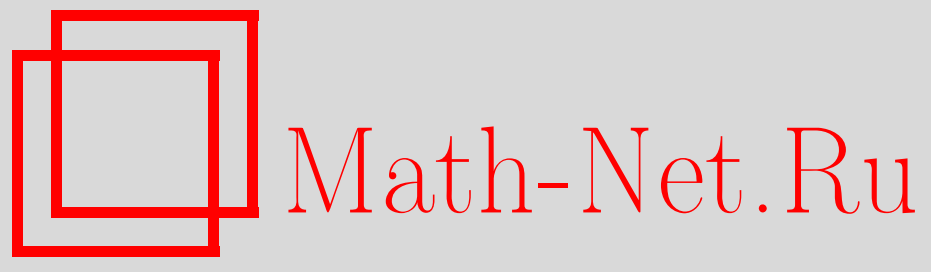

М. Э. Казарян, С. К. Ландо, Комбинаторные решения интегрируемых иерархий, УМН, 2015, том 70, выпуск $3,77-106$

DOI: https://doi.org/10.4213/rm9661

Использование Общероссийского математического портала Math-Net.Ru подразумевает, что вы прочитали и согласны с пользовательским соглашением http://www . mathnet.ru/rus/agreement

Параметры загрузки:

IP : 3.93.64.190

26 апреля 2023 г., 10:49:50

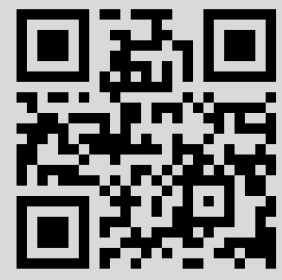




\section{Комбинаторные решения интегрируемых иерархий}

\section{М. Э. Казарян, С. К. Ландо}

В статье приводится обзор современных подходов к построению формальных решений интегрируемых иерархий математической физики, коэффициенты которых дают ответы в различных перечислительных задачах. Излагается связь этих подходов с комбинаторикой симметрических групп и их представлений. Описаны примеры применения полученных результатов к построению эффективных вычислений в задачах, связанных с моделями квантовых теорий поля.

Библиография: 34 названия.

Ключевые слова: интегрируемые системы, иерархия КадомцеваПетвиашвили, иерархия решетки Тоды, бозон-фермионное соответствие, числа Гурвица.

DOI: $10.4213 / \mathrm{rm} 9661$

\section{СОДЕРЖАНИЕ}

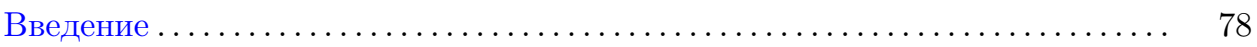

1. Иерархия Кадомцева-Петвиашвили ......................... 79

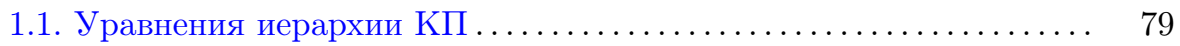

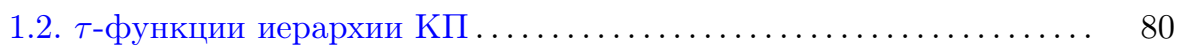

1.3. Вложения грассманианов и уравнения Плюккера ........... 81

1.4. Пространство рядов Лорана и его полубесконечная внешняя сте-

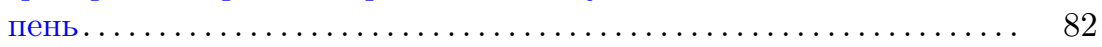

1.5. Бозон-фермионное соответствие .................... 82

1.6. Полубесконечный грассманиан и уравнения КП ............ 84

2. Комбинаторные представители семейства Орлова-Щербина решений

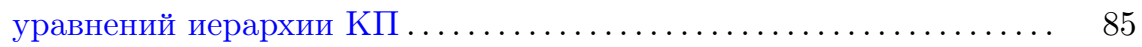

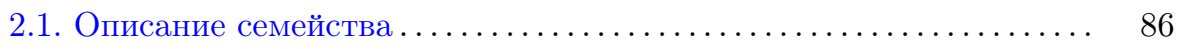

2.2. Формула характеров для чисел Гурвица................. 88

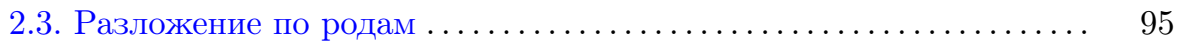

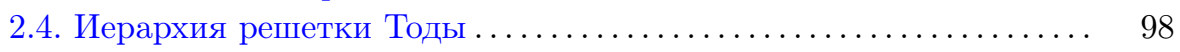

2.5. Приложения: перечисление карт и триангуляций............ 101

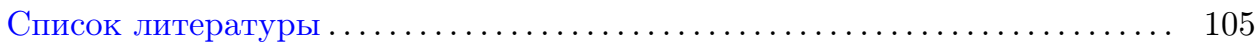

Работа выполнена при поддержке РФФИ (грант № 13-01-00383-а).

(C) М.Э. КАЗАРЯн, С.К. ЛАНДо, 2015 


\section{Введение}

Одна из моделей двумерной квантовой гравитации предполагает дискретизацию случайной метрической поверхности - ее представление в виде склейки одинаковых плоских правильных многоугольников суммарной площади 1. В частности, речь может идти о склейках треугольников (триангуляциях) или квадратов. Анализ такой модели требует исследования асимптотик различных числовых последовательностей комбинаторной природы, связанных со склейками (например, асимптотики числа триангуляций поверхности данного рода при стремлении числа треугольников к бесконечности). Несмотря на значительные усилия, приложенные в последние десятилетия как физиками, так и математиками к разработке общих перечислительных методов для решения подобных задач, применение разработанных методов не является автоматическим и до сих пор требует немалой изобретательности. В то же время в последние годы было осознано, что имеется большой класс перечислительных задач, ответом на которые служат производящие функции, представляющие собой решения интегрируемых иерархий математической физики.

О возможности возникновения решений интегрируемых иерархий (а именно, иерархии Кортевега-де Фриза) в подобных задачах известно уже более тридцати лет, однако имевшиеся конструкции предусматривали нетривиальное исследование особенностей производящих функций и переход к так называемому двойному скейлинговому пределу. Первым же естественным примером стала производящая функция для двойных чисел Гурвица. В 2000 г. Р. Пандхарипанде [26] высказал гипотезу о том, что эта функция является решением иерархии решетки Тоды, и А. Окуньков [23] в том же году ее доказал (см. п. 2.4). В свою очередь, интерес к изучению чисел Гурвица был обусловлен желанием исследовать геометрию пространств модулей комплексных кривых. Эта геометрия служит другой моделью двумерной квантовой гравитации, и гипотеза о появлении в ней решений иерархии Кортевега-де Фриза была высказана Виттеном [31] - с опорой на двойной скейлинговый предел. Результат Окунькова позволил авторам настоящей работы дать алгебро-геометрическое доказательство гипотезы Виттена [16].

После появления работы Окунькова естественные примеры комбинаторных решений интегрируемых иерархий стали множиться. Оказалось, что нелинейные уравнения в частных производных служат эффективными вычислительными инструментами для перечисления различных комбинаторных объектов, что, в свою очередь, дает эффективные методы вычисления различных асимптотических характеристик соответствующих последовательностей, ранее вычислению не поддававшихся.

Настоящая статья разбита на две части. В первой мы даем общее описание интегрируемой иерархии Кадомцева-Петвиашвили, которая может служить стандартным образцом подобных иерархий. Мы приводим принадлежащую Сато общую конструкцию решений этой иерархии.

Во второй части описано некоторое специальное бесконечномерное семейство решений иерархии Кадомцева-Петвиашвили и приведен целый ряд примеров перечисляющих производящих функций, принадлежащих этому семейству. Затем мы показываем, каким образом эти решения связаны с перечислением 
склеек многоугольников. Мы лишь упоминаем о связи построенных решений с геометрией пространств модулей алгебраических кривых - это отдельная обширная тема.

Авторы благодарны участникам семинара "Характеристические классы и теория пересечения" на факультете математики Высшей школы экономики за многочисленные плодотворные обсуждения.

\section{1. Иерархия Кадомцева-Петвиашвили}

Иерархия Кадомцева-Петвиашвили (ниже - КП) - это вполне интегрируемая система уравнений в частных производных на функции, зависящие от бесконечного набора переменных. Происхождение и физическая природа уравнений этой иерархии хорошо описаны, например, в [20], и мы не будем на них останавливаться. Нас будут интересовать лишь некоторые функции, удовлетворяющие всем уравнениям этой иерархии, и мы будем пользоваться описанием пространства ее решений, которое дадим ниже.

1.1. Уравнения иерархии КП. В математической физике имеются различные стандарты обозначения переменных в зависимости от выбора нормировки; мы будем пользоваться переменными $p_{1}, p_{2}, \ldots$, в которых младшее из уравнений иерархии КП имеет вид

$$
\frac{\partial^{2} F}{\partial p_{2}^{2}}=\frac{\partial^{2} F}{\partial p_{1} \partial p_{3}}-\frac{1}{2}\left(\frac{\partial^{2} F}{\partial p_{1}^{2}}\right)^{2}-\frac{1}{12} \frac{\partial^{4} F}{\partial p_{1}^{4}},
$$

или

$$
F_{2^{2}}=F_{1^{1} 3^{1}}-\frac{1}{2}\left(F_{1^{2}}\right)^{2}-\frac{1}{12} F_{1^{4}}
$$

(Здесь разбиение в индексе функции состоит из индексов переменных $p$, по которым ведется частное дифференцирование. Мы пользуемся мультипликативной записью разбиений; так, разбиение $2^{2} 3^{1}$ - это разбиение числа 7 , две части в котором равны 2, а третья равна 3.) В нем участвуют дифференцирования лишь по первым трем переменным, и сумма индексов в каждом мономе равна 4. В индексах следующих уравнений иерархии содержатся разбиения чисел $5,6, \ldots$ :

$$
\begin{aligned}
F_{2^{1} 3^{1}} & =-F_{1^{2}} F_{1^{1} 2^{1}}+F_{1^{1} 4^{1}}-\frac{1}{6} F_{1^{3} 2^{1}} \\
F_{2^{1} 4^{1}} & =-\frac{1}{2} F_{1^{1} 2^{1}}^{2}-F_{1^{2}} F_{1^{1} 3^{1}}+\frac{1}{8} F_{1^{3}}^{2}+\frac{1}{12} F_{1^{2}} F_{1^{4}}-\frac{1}{4} F_{1^{3} 3^{1}}+\frac{1}{120} F_{1^{6}}, \\
F_{3^{2}} & =\frac{1}{3} F_{1^{2}}^{3}-F_{1^{1} 2^{1}}^{2}-F_{1^{2}} F_{1^{1} 3^{1}}+F_{1^{1} 5^{1}}+\frac{1}{4} F_{1^{3}}^{2}+\frac{1}{3} F_{1^{2}} F_{1^{4}}-\frac{1}{3} F_{1^{3} 3^{1}}+\frac{1}{45} F_{1^{6}} .
\end{aligned}
$$

Отметим, что в левой части уравнений иерархии стоят вторые производные по переменным с номерами, большими 1, тогда как у производных в правой части номера всех переменных дифференцирования, кроме, быть может, одной, равны 1 . 
1.2. $\tau$-функции иерархии КП. Экспоненты решений иерархии КП называются ее $\tau$-функциями. Приведем пример $\tau$-функции (логарифм которой будет, разумеется, решением иерархии КП). Для задания ее коэффициентов нам потребуется следующее определение.

ОПРЕДЕЛЕНИЕ 1. Пусть $\mu$ - разбиение, $\mu \vdash|\mu|$. Простое число Гурвища $h_{m ; \mu}^{\circ}$ определяется равенством

$$
h_{m ; \mu}^{\circ}=\frac{1}{|\mu| !}\left|\left\{\left(\eta_{1}, \ldots, \eta_{m}\right), \eta_{i} \in C_{2}\left(S_{|\mu|}\right): \eta_{m} \circ \cdots \circ \eta_{1} \in C_{\mu}\left(S_{|\mu|}\right)\right\}\right| .
$$

Здесь $S_{|\mu|}$ - группа перестановок $|\mu|$ элементов, через $C_{2}\left(S_{|\mu|}\right)$ обозначено множество всех транспозиций в $S_{|\mu|}$, а $C_{\mu}\left(S_{|\mu|}\right)$ - это множество всех перестановок циклового типа $\mu \vdash|\mu|$ в $S_{|\mu|}$. (Цикловой тuп перестановки - это набор длин циклов в ее разложении в произведение независимых циклов; он образует разбиение числа переставляемых элементов. Все перестановки данного циклового типа образуют класс сопряженности элементов в группе перестановок.) В частности,

$$
C_{2}\left(S_{|\mu|}\right)=C_{1|\mu|-22^{1}}\left(S_{|\mu|}\right) .
$$

Другими словами, простые числа Гурвица перечисляют разложения данной перестановки в произведение данного числа транспозиций - в зависимости от циклового типа перестановки.

Связное простое число Гурвища $h_{m ; \mu}$ определяется аналогичным образом, но подсчитываются только такие последовательности из $m$ транспозиций, что порожденная ими подгруппа $\left\langle\eta_{1}, \ldots, \eta_{m}\right\rangle \subseteq S_{|\mu|}$ транзитивно действует на множестве $\{1, \ldots,|\mu|\}$.

Соберем простые числа Гурвица в две производящие функции:

$$
\begin{aligned}
H^{\circ}\left(u ; p_{1}, p_{2}, \ldots\right) & =\sum_{m=0}^{\infty} \sum_{\mu} h_{m ; \mu}^{\circ} p_{\mu_{1}} p_{\mu_{2}} \cdots \frac{u^{m}}{m !}, \\
H\left(u ; p_{1}, p_{2}, \ldots\right) & =\sum_{m=1}^{\infty} \sum_{\mu \neq \varnothing} h_{m ; \mu} p_{\mu_{1}} p_{\mu_{2}} \cdots \frac{u^{m}}{m !},
\end{aligned}
$$

где во втором случае $\mu$ пробегает множество всех разбиений всех натуральных чисел, а в первом к ним добавляется пустое разбиение (и ему соответствует слагаемое, равное 1). Эти производящие функции зависят от бесконечного числа переменных и являются формальными: на их сходимость не накладывается никаких условий. Из определений ясно, что их коэффициенты рациональны. Начальные члены разложений имеют следующий вид:

$$
\begin{aligned}
H^{\circ}\left(u ; p_{1}, p_{2}, \ldots\right)=1 & +p_{1}+\frac{p_{2} u}{2}+\frac{p_{1}^{2}}{2}+\frac{p_{3} u^{2}}{2}+\frac{1}{2} p_{2} p_{1} u+\frac{p_{1}^{3}}{6}+\frac{p_{2} u^{3}}{12} \\
& +\frac{2 p_{4} u^{3}}{3}+\frac{1}{4} p_{1}^{2} u^{2}+\frac{1}{2} p_{3} p_{1} u^{2}+\frac{1}{8} p_{2}^{2} u^{2}+\frac{1}{4} p_{2} p_{1}^{2} u+\frac{p_{1}^{4}}{24}+\cdots \\
H\left(u ; p_{1}, p_{2}, \ldots\right)=p_{1} & +\frac{p_{2} u}{2}+\left(\frac{p_{1}^{2}}{4}+\frac{p_{3}}{2}\right) u^{2}+\left(\frac{2 p_{1} p_{2}}{3}+\frac{p_{2}}{12}+\frac{2 p_{4}}{3}\right) u^{3} \\
& +\left(\frac{p_{1}^{3}}{6}+\frac{p_{1}^{2}}{48}+\frac{9 p_{3} p_{1}}{8}+\frac{p_{2}^{2}}{2}+\frac{3 p_{3}}{8}+\frac{25 p_{5}}{24}\right) u^{4}+\cdots
\end{aligned}
$$


Весьма общее комбинаторное утверждение о перечислении связных и несвязных объектов дает следующее соотношение:

$$
H^{\circ}=\exp \{H\} .
$$

В результате утверждения про простые и связные простые числа Гурвица можно друг в друга переформулировать.

Теорема 1.1 [23], [16]. Производящая функиия $H^{\circ}\left(u ; p_{1}, p_{2}, \ldots\right)$ для простых чисел Гурвица является однопараметрическим семейством $\tau$-функиий для иерархии KП, а ее логарифм $H\left(u ; p_{1}, p_{2}, \ldots\right)$ является однопараметрическим семейством решений этой иерархии.

Описываемая нами ниже конструкция Сато [28] интерпретирует пространство решений уравнений КП как грассманиан полубесконечных плоскостей в бесконечномерном пространстве. Она позволяет построить все решения уравнений КП. Для решения обратной задачи - доказать, что данная функция является решением - достаточно указать соответствующую ей полубесконечную плоскость. Это означает, в частности, что для идентификации функции в качестве решения иерархии КП нет необходимости знать уравнения иерархии. Мы укажем, в частности, и полубесконечную плоскость, отвечающую производящей функции $H$.

1.3. Вложения грассманианов и уравнения Плюккера. Рассмотрим грассманиан $G(2, V)$ двумерных плоскостей в четырехмерном векторном пространстве $V \equiv \mathbb{C}^{4}$. Всякую двумерную плоскость в $V$ можно представить в виде внешнего произведения $\beta_{1} \wedge \beta_{2}$ любой пары принадлежащих ей линейно независимых векторов $\beta_{1}, \beta_{2}$. Это векторное произведение определено корректно с точностью до ненулевого множителя; оно определяет двумерную плоскость однозначно, а значит, таким образом можно задать вложение грассманиана $G(2, V)$ в проективизацию внешнего квадрата пространства $V$ : $G(2, V) \hookrightarrow P \Lambda^{2} V$. Изложенная конструкция немедленно обобщается на вложения произвольного грассманиана $G(k, V) \quad k$-мерных плоскостей в $n$-мерном векторном пространстве $V$ в проективизацию $P \Lambda^{k} V$.

Уравнения Плюккера - это уравнения, задающие образ указанного вложения. Грассманиан $G(k, V)$ при $\operatorname{dim} V=n$ имеет размерность $k(n-k)$, а размерность пространства $P \Lambda^{k} V$ равна $\left(\begin{array}{l}n \\ k\end{array}\right)-1$, что, как правило, превосходит $k(n-k)$; значит, образ плюккерова вложения, вообще говоря, не совпадает со всем проективизированным внешним произведением $P \Lambda^{k} V$. Например, образ вложения многообразия $G(2,4)$ в $P \Lambda^{2} \mathbb{C}^{4}$ представляет собой гиперповерхность в пятимерном проективном пространстве.

Для того чтобы задать эту гиперповерхность уравнением, выберем базис $e_{1}$, $e_{2}, e_{3}, e_{4}$ в $V$. Этот базис порождает базис $\beta_{i j}=e_{i} \wedge e_{j}, 1 \leqslant i<j \leqslant 4$, пространства $\Lambda^{2} V$ и естественную систему координат $y_{i j}$ в этом пространстве. Образ вложения грассманиана состоит из разложимых векторов. По определению внешнего произведения образ плоскости, натянутой на пару векторов $\left(a_{1}, a_{2}, a_{3}, a_{4}\right),\left(b_{1}, b_{2}, b_{3}, b_{4}\right)$, имеет проективные координаты

$$
y_{i j}=\left|\begin{array}{cc}
a_{i} & b_{i} \\
a_{j} & b_{j}
\end{array}\right|=a_{i} b_{j}-a_{j} b_{i},
$$


откуда следует, что

$$
y_{12} y_{34}-y_{13} y_{24}+y_{14} y_{23}=0 .
$$

Это и есть уравнение Плюккера вложения.

Для произвольных значений $n$ и $k$ уравнения Плюккера остаются квадратичными. Другими словами, идеал в кольце многочленов, состоящий из многочленов, обращающихся в нуль на образе плюккерова вложения, порождается квадратичными многочленами (см., например, [9]).

\section{4. Пространство рядов Лорана и его полубесконечная внешняя} степень. Возьмем в качестве пространства $V$ бесконечномерное пространство рядов Лорана от одной переменной. Элементы этого пространства имеют вид

$$
c_{-k} z^{-k}+c_{-k+1} z^{-k+1}+\cdots
$$

Мономы $z^{k}, k=\ldots,-2,-1,0,1,2, \ldots$, образуют стандартный базис в $V$. По определению, полубесконечная внешняя степенъ $\Lambda^{\infty / 2} V$ - это векторное пространство, натянутое на векторы

$$
v_{\mu}=z^{m_{1}} \wedge z^{m_{2}} \wedge z^{m_{3}} \wedge \cdots, \quad m_{1}<m_{2}<m_{3}<\cdots, \quad m_{i}=\mu_{i}-i,
$$

где $\mu$ - разбиение, $\mu=\left(\mu_{1}, \mu_{2}, \mu_{3}, \ldots\right), \mu_{1} \geqslant \mu_{2} \geqslant \mu_{3} \geqslant \cdots$, в котором все части, за исключением конечного числа, равны 0 . В частности, $m_{i}=-i$ для всех достаточно больших $i$.

Пустому разбиению отвечает вакуум-вектор

$$
v_{\varnothing}=z^{-1} \wedge z^{-2} \wedge z^{-3} \wedge \cdots .
$$

Аналогично,

$$
\begin{aligned}
& v_{1^{1}}=z^{0} \wedge z^{-2} \wedge z^{-3} \wedge \cdots, \\
& v_{2^{1}}=z^{1} \wedge z^{-2} \wedge z^{-3} \wedge \cdots, \\
& v_{1^{2}}=z^{0} \wedge z^{-1} \wedge z^{-3} \wedge \cdots \quad \text { ит. д. }
\end{aligned}
$$

1.5. Бозон-фермионное соответствие. Нумерация базисных векторов полубесконечного внешнего произведения $\Lambda^{\infty / 2} V$ (пространства фермионов) разбиениями целых неотрицательных чисел устанавливает его естественный изоморфизм с пространством бозонов - степенных рядов от бесконечного набора переменных $p_{1}, p_{2}, \ldots$ - как векторных пространств. Этот изоморфизм переводит базисный вектор $v_{\mu}$ в многочлен Шура $s_{\mu}=s_{\mu}\left(p_{1}, p_{2}, \ldots\right)$. Последний является квазиоднородным многочленом степени $|\mu|$ от переменных $p_{i}$ (степень переменной $p_{i}$ по определению равна $i$ ). Многочлены Шура образуют аддитивный базис векторного пространства степенных рядов от переменных $p_{i}$.

Многочлен Шура $s_{k}$, отвечающий одночастичному разбиению $k^{1} \vdash k$, определяется с помощью разложения

$$
\begin{gathered}
s_{0}+s_{1} z+s_{2} z^{2}+s_{3} z^{3}+s_{4} z^{4}+\cdots=\exp \left\{p_{1} z+p_{2} \frac{z^{2}}{2}+p_{3} \frac{z^{3}}{3}+\cdots\right\} \\
=1+p_{1} z+\frac{1}{2}\left(p_{1}^{2}+p_{2}\right) z^{2}+\frac{1}{6}\left(p_{1}^{3}+3 p_{1} p_{2}+2 p_{3}\right) z^{3}+\cdots
\end{gathered}
$$


Для произвольного разбиения $\kappa=\left(\kappa_{1}, \kappa_{2}, \kappa_{3}, \ldots\right), \kappa_{1} \geqslant \kappa_{2} \geqslant \kappa_{3} \geqslant \cdots$, многочлен Шура $s_{\kappa}$ представляет собой определитель:

$$
s_{\kappa}=\operatorname{det}\left\|s_{\kappa_{j}-j+i}\right\| \text {. }
$$

Здесь индексы $i, j$ пробегают множество $\{1,2, \ldots, n\}$ для достаточно большого $n$, и поскольку при достаточно больших $i$ имеем $\kappa_{i}=0$, этот определитель, а значит, и многочлен $s_{\kappa}$, не зависит от $n$. Так, например,

$$
\begin{aligned}
& s_{1^{3}}=\left|\begin{array}{ccccc}
s_{1} & s_{2} & s_{3} & s_{4} & \ldots \\
s_{0} & s_{1} & s_{2} & s_{3} & \ldots \\
0 & s_{0} & s_{1} & s_{2} & \ldots \\
0 & 0 & 0 & s_{0} & \ldots \\
\ldots \ldots & \ldots & \ldots
\end{array}\right| \\
& =\left|\begin{array}{cccc}
p_{1} & \frac{1}{2}\left(p_{1}^{2}+p_{2}\right) & \frac{1}{6}\left(p_{1}^{3}+3 p_{1} p_{2}+2 p_{3}\right) & \ldots \\
1 & p_{1} & \frac{1}{2}\left(p_{1}^{2}+p_{2}\right) & \ldots \\
0 & 1 & p_{1} & \frac{1}{2}\left(p_{1}^{2}+p_{2}\right) \\
0 & 0 & 0 & 1 \\
\ldots \ldots \ldots \ldots \ldots \ldots \ldots \ldots \ldots \ldots \ldots \ldots \ldots \ldots \ldots \ldots \ldots \ldots \ldots \ldots \ldots \ldots
\end{array}\right| \\
& =\left|\begin{array}{ccc}
p_{1} & \frac{1}{2}\left(p_{1}^{2}+p_{2}\right) & \frac{1}{6}\left(p_{1}^{3}+3 p_{1} p_{2}+2 p_{3}\right) \\
1 & p_{1} & \frac{1}{2}\left(p_{1}^{2}+p_{2}\right) \\
0 & 1 & p_{1}
\end{array}\right| .
\end{aligned}
$$

Вот несколько первых многочленов Шура:

$$
\begin{array}{ll}
s_{0}=1, \quad s_{1^{1}}=p_{1}, & s_{2^{1}}=\frac{1}{2}\left(p_{1}^{2}+p_{2}\right), \quad s_{3^{1}}=\frac{1}{6}\left(p_{1}^{3}+3 p_{1} p_{2}+2 p_{3}\right), \\
s_{1^{2}}=\frac{1}{2}\left(p_{1}^{2}-p_{2}\right), \quad s_{1^{1} 2^{1}}=\frac{1}{3}\left(p_{1}^{3}-p_{3}\right), & s_{1^{3}}=\frac{1}{6}\left(p_{1}^{3}-3 p_{1} p_{2}+2 p_{3}\right) .
\end{array}
$$

Многочлены Шура играют ключевую роль в изучении представлений симметрических групп, таким образом, в их появлении в задачах перечисления разложений перестановок в произведение нет ничего удивительного.

Есть и другой, более инвариантный, способ описать бозон-фермионное соответствие. А именно, для $i=1,2,3, \ldots$ обозначим через $\widehat{z^{-i}}$ оператор сдвига, действующий в пространстве $V$ и переводящий $z^{m}$ в $z^{m-i}, m \in \mathbb{Z}$. Это действие продолжается на пространство фермионов по правилу Лейбница:

$$
\widehat{z^{-i}}: z^{m_{1}} \wedge z^{m_{2}} \wedge \cdots \mapsto z^{m_{1}-i} \wedge z^{m_{2}} \wedge \cdots+z^{m_{1}} \wedge z^{m_{2}-i} \wedge \cdots+\cdots
$$

Обозначим треугольными скобками $\langle v\rangle$ коэффициент при вакуум-векторе $v_{\varnothing}$ в разложении фермиона $v$ по базису $v_{\mu}$. В этих обозначениях бозон-фермионное соответствие сопоставляет вектору $v \in \Lambda^{\infty / 2} V$ следующий формальный ряд 
от переменных $p_{k}$ :

$$
v \mapsto\left\langle\exp \left\{\sum_{i=1}^{\infty} \frac{p_{i}}{i} \widehat{z^{-i}}\right\} v\right\rangle .
$$

Эта формула интерпретируется следующим образом. При бозон-фермионном соответствии оператор $\widehat{z^{-i}}$, действующий в пространстве фермионов, переходит в оператор $i \partial / \partial p_{i}$, действующий в пространстве бозонов. Так что приведенная формула есть не что иное, как формула Тейлора

$$
f(p)=\left.\exp \left\{\sum_{i=0}^{\infty} p_{i} \frac{\partial}{\partial q_{i}}\right\} f(q)\right|_{q=0} .
$$

Отметим также, что для положительных $i$ оператор сдвига $\widehat{z^{i}}$ действует в пространстве бозонов умножением на переменную $p_{i}$. Поэтому в обратную сторону бозон-фермионное соответствие сопоставляет многочлену (или, более общим образом, степенному ряду) $f\left(p_{1}, p_{2}, \ldots\right)$ вектор $f\left(\widehat{z^{1}}, \widehat{z^{2}}, \ldots\right) v_{\varnothing}$ из пространства фермионов.

1.6. Полубесконечный грассманиан и уравнения КП. Полубесконечный грассманиан $G(\infty / 2, V)$ состоит из разложимых векторов в $P \Lambda^{\infty / 2} V$, т. е. из векторов вида

$$
\beta_{1}(z) \wedge \beta_{2}(z) \wedge \beta_{3}(z) \wedge \cdots,
$$

где $\beta_{i}$ - это ряды Лорана от переменной $z$ и для достаточно больших $i$ старший член разложения ряда $\beta_{i}$ равен $z^{-i}$ :

$$
\beta_{i}(z)=z^{-i}+c_{i 1} z^{-i+1}+c_{i 2} z^{-i+2}+\cdots .
$$

Каждое такое бесконечное внешнее произведение, рассматриваемое с точностью до умножения на ненулевую константу, взаимно однозначно соответствует полубесконечной плоскости в $V$, натянутой на векторы $\beta_{1}, \beta_{2}, \ldots$.

ОПРедЕЛЕНиЕ 2. Уравнения Хироты - это уравнения Плюккера вложения полубесконечного грассманиана в проективизированное полубесконечное внешнее произведение $P \Lambda^{\infty / 2} V$. Решения уравнений Хироты (т. е. полубесконечные плоскости) называются $\tau$-функииями иерархии КП.

Вот один из способов представить уравнения Хироты: тождественно по $p$ и $q$ должно выполняться равенство

$$
\begin{aligned}
& \operatorname{Res}_{z=0} \exp \left\{2 \sum_{i=0}^{\infty} \frac{q_{i}}{i} z^{-i}\right\} \\
& \times \tau\left(p_{1}-q_{1}+z, p_{2}-q_{2}+z^{2}, \ldots\right) \tau\left(p_{1}+q_{1}-z, p_{2}+q_{2}-z^{2}, \ldots\right) \frac{d z}{z^{2}}=0
\end{aligned}
$$

здесь через $\operatorname{Res}_{z=0}$ обозначена операция взятия вычета 1-формы переменной $z$ в точке $z=0$.

Приведенное равенство интерпретируется следующим образом. Разложим его левую часть в формальный ряд по $q$-переменным. Тогда коэффициент при 
каждом мономе является конечной квадратичной полиномиальной комбинацией частных производных функции $\tau=\tau\left(p_{1}, p_{2}, \ldots\right)$. Например, уравнение, соответствующее моному $q_{3}$, имеет вид

$$
\tau_{2}^{2}-\tau_{1} \tau_{3}-\frac{1}{4} \tau_{1^{2}}^{2}+\tau_{1} \tau_{3}-\tau \tau_{2^{2}}+\frac{1}{3} \tau_{1} \tau_{1^{3}}-\frac{1}{12} \tau \tau_{1^{4}}=0
$$

Каждое из таких квадратичных дифференциальных уравнений можно рассматривать как совокупность квадратичных алгебраических соотношений на коэффициенты Тейлора функции $\tau$, которые и образуют соотношения Плюккера. С другой стороны, после подстановки $\tau=e^{F}$ уравнения на функцию $\tau$ преобразуются в уравнения на функцию $F$. Например, приведенное выше уравнение на функцию $\tau$ эквивалентно уравнению (1).

ОПРЕДЕЛЕНИЕ 3. Форма, которую принимают уравнения Хироты как уравнения на логарифм $\tau$-функции, называется уравнениями иерархии Кадомцева-Петвиашвили.

Другими словами, всякое решение иерархии КП можно получить в результате следующей процедуры:

- возьмем внешнее произведение $\beta_{1}(z) \wedge \beta_{2}(z) \wedge \cdots$, соответствующее какой-либо полубесконечной плоскости в пространстве $V$;

- воспользовавшись разложениями функций $\beta_{i}$ в ряды Лорана, представим соответствующую точку в полубесконечном грассманиане в виде линейной комбинации базисных векторов $v_{\kappa}$ (содержащей, вообще говоря, бесконечно много ненулевых слагаемых) и умножим на константу, чтобы сделать коэффициент при вакуум-векторе $v_{\varnothing}$ равным 1 ;

- заменим в получившейся линейной комбинации всякий вектор $v_{\kappa}$ соответствующим многочленом Шура $s_{\kappa}\left(p_{1}, p_{2}, \ldots\right)$, получив тем самым степенной ряд от бесконечного набора переменных $p_{1}, p_{2}, \ldots$;

- возьмем логарифм полученного ряда.

Таким образом, в наших руках имеется универсальный механизм, позволяющий генерировать все решения иерархии КП. Задача же проверки того, является ли та или иная функция таким решением, как правило, более сложна. Полубесконечную плоскость, отвечающую производящей функции $H^{\circ}$ для простых чисел Гурвица, мы предъявим в п. 2.2 .

\section{2. Комбинаторные представители семейства Орлова-Щербина решений уравнений иерархии КП}

Производящая функция для чисел Гурвица принадлежит одному довольно обширному семейству решений иерархии КП. Упомянутое семейство содержит большое количество других комбинаторных решений иерархии, коэффициенты которых перечисляют различные объекты, связанные с комбинаторикой симметрических групп. В этом разделе мы дадим описание этого семейства и приведем различные специализации его параметров, приводящие к ответам в некоторых комбинаторных задачах. 
2.1. Описание семейства. Каждому разбиению $\mu=\left(\mu_{1}, \ldots, \mu_{\ell}\right)$ числа $n$ можно стандартным образом поставить в соответствие диаграмму Юнга, состоящую из $n$ клеток; количество строчек $\ell$ в этой диаграмме, т. е. количество частей в разбиении $\mu$, мы будет обозначать через $\ell(\mu)$. Назовем содержанием клетки $w$ диаграммы Юнга, находящейся на пересечении ее $i$-й строки и $j$-го столбца, величину

$$
c(w)=j-i, \quad 1 \leqslant i \leqslant \ell(\mu), \quad 1 \leqslant j \leqslant \mu_{i} .
$$

Содержанием $c(\mu)$ диаграммы $\mu$ будем называть неупорядоченный набор содержаний ее клеток. Положим также

$$
y_{\mu}=\prod_{w \in \mu} y_{c(w)} .
$$

Например,

$$
c((5,3,3,2))=(-3,-2,-2,-1,-1,0,0,0,1,1,2,3,4)
$$

и

$$
y_{(5,3,3,2)}=y_{-3} y_{-2}^{2} y_{-1}^{2} y_{0}^{3} y_{1}^{2} y_{2} y_{3} y_{4}
$$

степень этого монома равна количеству клеток в диаграмме (в данном примере клеток 13, см. рис. 1).

\begin{tabular}{|c|c|c|c|c|}
\hline 0 & 1 & 2 & 3 & 4 \\
\hline-1 & 0 & 1 & \multicolumn{2}{|c}{} \\
\hline-2 & -1 & 0 & \multicolumn{2}{|}{} \\
\cline { 1 - 2 }-3 & -2 & \multicolumn{2}{|l}{} \\
\cline { 1 - 2 } & &
\end{tabular}

Рис. 1. Диаграмма Юнга, в каждой клетке которой записано содержание этой клетки

Следующая теорема представляет собой результат А. Ю. Орлова и Д. М. Щербина [25] в форме, которую придали ему Гульден и Джексон [8].

Теорема 2.1. Производящая функиия

$$
\sum_{\mu} y_{\mu} \frac{\operatorname{dim}_{\mu}}{|\mu| !} s_{\mu}\left(p_{1}, p_{2}, \ldots\right),
$$

где $\operatorname{dim}_{\mu}$ есть размерность неприводимого представления симметрической группы $S_{|\mu|}$, отвечающего разбиению $\mu$, является семейством $\tau$-функиий иерархии КП.

Отметим, что множитель $\operatorname{dim}_{\mu} /|\mu|$ ! может быть записан в виде

$$
\frac{\operatorname{dim}_{\mu}}{|\mu| !}=s_{\mu}(1,0,0, \ldots) .
$$

В частности, подставляя вместо переменных $\ldots, y_{-2}, y_{-1}, y_{0}, y_{1}, y_{2}, \ldots$ конкретные значения, мы получаем конкретные $\tau$-функции, а их логарифмы дают решения иерархии КП. 
ДоказАтельство. При $y_{c} \equiv 1, c=\ldots,-2,-1,0,1,2, \ldots$, указанная $\tau$-функция принимает вид

$$
\sum_{\mu} \frac{\operatorname{dim}_{\mu}}{|\mu| !} s_{\mu}(p)=e^{p_{1}}
$$

что является специализацией хорошо известного тождества для функций Шура:

$$
\exp \left\{\sum_{k=1}^{\infty} \frac{p_{k} q_{k}}{k}\right\}=\sum_{\mu} s_{\mu}(p) s_{\mu}(q)
$$

(см., например, [27]). Эта $\tau$-функция соответствует в фермионном представлении бесконечному произведению $\beta_{1} \wedge \beta_{2} \wedge \beta_{3} \wedge \cdots$, в котором

$$
\beta_{k}=e^{z} z^{-k}=\sum_{i=0}^{\infty} \frac{z^{i-k}}{i !}
$$

Заметим, что ее логарифм $p_{1}$ является решением иерархии КП по очевидной причине - все производные в уравнениях иерархии имеют по крайней мере второй порядок.

В случае общих значений параметров $y_{c}$ предъявленное фермионное представление нужно модифицировать следующим образом. Положим

$$
u_{k}= \begin{cases}\prod_{i=1}^{k} y_{i}, & k>0 \\ \left(\prod_{i=k+1}^{0} y_{i}\right)^{-1}, & k \leqslant 0\end{cases}
$$

Иными словами, мы выбрали $u_{k}$ так, чтобы при всех целых $k \leqslant \ell$ выполнялось равенство $\frac{u_{k}}{u_{\ell}}=\prod_{i=k+1}^{\ell} y_{i}$. В этих обозначениях компоненты внешнего произведения, соответствующего $\tau$-функции теоремы, имеют вид

$$
\beta_{k}=\frac{1}{u_{-k}} \sum_{i=0}^{\infty} u_{i-k} \frac{z^{i-k}}{i !} .
$$

Действительно, при такой модификации внешнего произведения его коэффициент при $v_{\mu}=z^{\mu_{1}-1} \wedge z^{\mu_{2}-2} \wedge \cdots$ в разложении по базисным векторам умножится на моном $\prod_{k=1}^{\infty} \frac{u_{\mu_{k}-k}}{u_{-k}}=y_{\mu}$, чего мы и добивались. Теорема доказана.

ЗАмечАнИЕ 2.2. Действуя на полубесконечную плоскость в пространстве рядов Лорана $V$ линейным оператором, мы получаем, вообе говоря, другую полубесконечную плоскость, которая, в свою очередь, задает другую $\tau$-функцию иерархии КП. В частности, $\tau$-функции семейства Орлова-Щербина получаются из $\tau$-функции $e^{p_{1}}$ в результате действия на плоскость (5) диагональным 
оператором, матрица которого в стандартном базисе имеет вид

$$
\left(\begin{array}{cccccccc}
\ddots & \ldots & \ldots & \ldots & \ldots & \ldots & \ldots & \ldots \\
\cdots & \frac{1}{y_{0} y_{-1} y_{-2}} & 0 & 0 & 0 & 0 & 0 & \ldots \\
\cdots & 0 & \frac{1}{y_{0} y_{-1}} & 0 & 0 & 0 & 0 & \ldots \\
\cdots & 0 & 0 & \frac{1}{y_{0}} & 0 & 0 & 0 & \ldots \\
\cdots & 0 & 0 & 0 & 1 & 0 & 0 & \ldots \\
\cdots & 0 & 0 & 0 & 0 & y_{1} & 0 & \ldots \\
\cdots & 0 & 0 & 0 & 0 & 0 & y_{1} y_{2} & \ldots \\
\ldots & \ldots & \ldots & \ldots & \ldots & \ldots & \ldots & \ddots
\end{array}\right) .
$$

ПримеР 2.3. Чтобы получить $\tau$-функцию $H^{\circ}$ для простых чисел Гурвица, нужно положить $y_{c}=e^{u c}, c=\ldots,-2,-1,0,1,2, \ldots$. Доказательство этого факта приведено ниже, в п. 2.2 .

2.2. Формула характеров для чисел Гурвица. Как мы уже обсуждали, числа Гурвица определяются в терминах комбинаторики симметрической группы. Рассмотрим это соответствие более подробно. Пусть $S_{n}$ - группа перестановок из $n$ элементов, $\mathbb{C} S_{n}$ - ее групповая алгебра, $Z \mathbb{C} S_{n}-$ центр групповой алгебры. Для всякого разбиения $\mu$ числа $n$ обозначим через $C_{\mu} \in Z \mathbb{C} S_{n}$ сумму перестановок циклового типа $\mu$. Все перестановки данного циклового типа $\mu$ сопряжены друг с другом и образуют класс сопряженности; через $\left|C_{\mu}\right|$ будет обозначаться количество элементов в этом классе. Элементы $C_{\mu} /\left|C_{\mu}\right|$ образуют аддитивный базис в пространстве $Z \mathbb{C} S_{n}$. По определению, число Гурвица $h_{m ; \mu}^{\circ}$ - это коэффициент при $C_{\mu} /\left|C_{\mu}\right|$ в разложении элемента $\left(C_{2}\right)^{m} / n$ ! по этому базису, где $C_{2}=C_{1^{n-2} 2^{1}}$ - сумма транспозиций.

Для вычисления элемента $\left(C_{2}\right)^{m}$ и вообще для вычислений в алгебре $Z \mathbb{C} S_{n}$ удобнее, однако, пользоваться другим базисом, состоящим из элементов $F_{\lambda}$, образующих систему идемпотентов, $F_{\lambda} F_{\mu}=\delta_{\lambda, \mu} F_{\lambda}$. По определению, $F_{\lambda} \in$ $Z \mathbb{C} S_{n}$ - это элемент, который действует тождественно в неприводимом представлении $\lambda$ и тривиально во всех остальных неприводимых представлениях группы $S_{n}$. Напомним, что всякий элемент центра действует в каждом неприводимом представлении скалярным оператором. Отсюда вытекает, что оператор умножения на любой элемент $a \in Z \mathbb{C} S_{n}$ в базисе $F_{\lambda}$ оказывается диагональным:

$$
a=\sum_{\lambda \vdash n} f_{a}(\lambda) F_{\lambda}, \quad a F_{\lambda}=f_{a}(\lambda) F_{\lambda} .
$$

Число $f_{a}(\lambda)$ - это также значение той константы, умножением на которую элемент $a \in Z \mathbb{C} S_{n}$ действует в неприводимом представлении $\lambda$. Из теории характеров вытекает следующее полезное тождество.

ПРЕДЛОЖЕНИЕ 2.4. Для всякого элемента

$$
a=\sum_{\mu \vdash n} a_{\mu} \frac{C_{\mu}}{\left|C_{\mu}\right|} \in Z \mathbb{C} S_{n}, \quad a_{\mu} \in \mathbb{C},
$$


имеет место следующее равенство (в пространстве многочленов от переменных $\left.p_{1}, p_{2}, \ldots\right)$ :

$$
\sum_{\mu \vdash n} a_{\mu} p_{\mu_{1}} p_{\mu_{2}} \cdots=\sum_{\mu \vdash n} f_{a}(\mu) \operatorname{dim}_{\mu} s_{\mu}(p),
$$

где $f_{a}(\mu)$ - собственное значение оператора умножения на а, отвечающее базисному собственному вектору $F_{\mu}$.

Это равенство часто используется в качестве определения функций Шура. Отметим, что при стандартной интерпретации функции Шура как симметрической функции от некоторого набора образующих $x_{1}, \ldots, x_{N}$ переменные $p_{k}$ соответствуют сумме $k$-х степеней этих образующих: $p_{k}=x_{1}^{k}+\cdots+x_{N}^{k}$.

В частности, применяя это утверждение к определению чисел Гурвица, мы получаем равенство

$$
H^{\circ}\left(u ; p_{1}, p_{2}, \ldots\right)=\sum_{\lambda} e^{f_{2}(\lambda) u} \frac{\operatorname{dim}_{\mu}}{|\mu| !} s_{\lambda}(p),
$$

где $f_{2}(\lambda)=f_{1^{n-2} 2^{1}}(\lambda)$ - соответствующее собственное число оператора умножения на сумму транспозиций.

Чтобы полностью идентифицировать функцию $H^{\circ}$, нам осталось вычислить собственные числа $f_{2}(\lambda)$. Это удобно делать с помощью формализма, основанного на использовании элементов Юциса-Мерфи.

ОПРеДЕЛЕНИЕ 4. Элементами Юииса-Мерфи групповой алгебры $\mathbb{C} S_{n}$ называются следующие суммы транспозиций:

$$
X_{1}=0, \quad X_{2}=(1,2), \quad X_{3}=(1,3)+(2,3), \quad X_{4}=(1,4)+(2,4)+(3,4), \quad \ldots,
$$

T. e.

$$
X_{k}=\sum_{i=1}^{k-1}(i, k), \quad k=1,2, \ldots, n .
$$

Эти элементы не лежат в центре групповой алгебры, однако имеют место следующие утверждения.

ПРЕДЛОЖЕНИЕ 2.5 [14], [21], [30]. 1. Элементы $X_{1}, X_{2}, \ldots, X_{n}$ коммутируют между собой и тем самым порождают коммутативную подалгебру в $\mathbb{C} S_{n}$.

2. Симметрические многочлены от элементов $X_{k}$ лежат в центре $Z \mathbb{C} S_{n}$, причем всякий элемент иентра может быть представлен как симметрический многочлен от элементов Юииса-Мерфи.

3. Если некоторый элемент $a \in Z \mathbb{C} S_{n}$ представлен как симметрический многочлен от элементов Ючиса-Мерфи: $a=P_{a}\left(X_{1}, \ldots, X_{n}\right)$, то собственное число $f_{a}(\lambda)$ действия элемента а в представлении $\lambda$ равно значению симметрической функиии $P$ на содержании с $(\lambda)$ диаграммы Юнга $\lambda$ :

$$
P_{a}\left(X_{1}, \ldots, X_{n}\right) F_{\lambda}=P(c(\lambda)) F_{\lambda} .
$$


Например, для элемента $C_{2}=\sum_{k=1}^{n} X_{k}$ собственное число, отвечающее диа-
Юамме Юнга $\lambda$, равно

$$
f_{2}(\lambda)=\sum_{w \in \lambda} c(w)=\frac{1}{2} \sum_{i=1}^{\ell(\lambda)}\left(\left(\lambda_{i}-i+\frac{1}{2}\right)^{2}-\left(-i+\frac{1}{2}\right)^{2}\right) .
$$

Соответственно, производящая функция $H^{\circ}$ записывается в виде элемента семейства Орлова-Щербина с $y_{c}=e^{c u}$, что и доказывает утверждение примеpa 2.3 .

ПримеР 2.6 (обобщенные числа Гурвица). Простые числа Гурвица перечисляют разложения перестановки данного циклового типа в произведение данного числа транспозиций. Рассмотрим более общую задачу, в которой транспозиции заменяются на перестановки с фиксированным количеством циклов. Эти числа были введены в [8].

Назовем вырожденностъю разбиения $\lambda=\left(\lambda_{1}, \ldots, \lambda_{\ell}\right)$ число

$$
k(\lambda)=|\lambda|-\ell(\lambda)=\sum_{i=1}^{\ell}\left(\lambda_{i}-1\right) .
$$

Вырожденностью перестановки называется вырожденность набора длин ее циклов. Иначе говоря, вырожденность перестановки - минимальное число транспозиций, в произведение которых эта перестановка может быть разложена. Положим

$$
a_{k_{1}, \ldots, k_{m} ; \mu}^{\circ}=\frac{1}{|\mu| !}\left|\left\{\left(\tau_{1}, \ldots, \tau_{m}\right), \tau_{i} \in S_{|\mu|}: k\left(\tau_{i}\right)=k_{i}, \tau_{1} \circ \cdots \circ \tau_{m} \in C_{\mu}\left(S_{|\mu|}\right)\right\}\right|
$$

и определим числа $a_{k_{1}, \ldots, k_{m} ; \mu}$ аналогичным образом, потребовав дополнительно, чтобы подгруппа в $S_{|\mu|}$, порожденная перестановками $\tau_{1}, \ldots, \tau_{\mu}$, действовала на множестве $\{1, \ldots,|\mu|\}$ транзитивно. Производящие функции

$$
A_{m}^{\circ}\left(u_{1}, \ldots, u_{m} ; p_{1}, p_{2}, \ldots\right)=\sum_{\mu, k_{1}, \ldots, k_{m}} a_{k_{1}, \ldots, k_{m} ; \mu}^{\circ} u_{1}^{k_{1}} \cdots u_{m}^{k_{m}} p_{\mu_{1}} p_{\mu_{2}} \ldots
$$

и

$$
A_{m}\left(u_{1}, \ldots, u_{m} ; p_{1}, p_{2}, \ldots\right)=\sum_{\mu, k_{1}, \ldots, k_{m}} a_{k_{1}, \ldots, k_{m} ; \mu} u_{1}^{k_{1}} \cdots u_{m}^{k_{m}} p_{\mu_{1}} p_{\mu_{2}} \ldots
$$

связаны между собой соотношением

$$
A_{m}^{\circ}=\exp \left\{A_{m}\right\}
$$

и справедливо следующее утверждение.

Теорема 2.7 [8]. Производящая функиия $A_{m}^{\circ}\left(u_{1}, \ldots, u_{m} ; p_{1}, p_{2}, \ldots\right)$ для обобщенных чисел Гурвица является т-параметрическим семейством $\tau$-функицй иерархии $K \Pi$, а ее логарифм $A_{m}\left(u_{1}, \ldots, u_{m} ; p_{1}, p_{2}, \ldots\right)$ является $m$-параметрическим семейством решений этой иерархии. 
Производящая функция $A_{m}^{\circ}$ соответствует набору значений параметров

$$
y_{c}=\prod_{i=1}^{m}\left(1+u_{i} c\right) \quad \text { для } \quad c=\ldots,-2,-1,0,1,2, \ldots
$$

в семействе Орлова-Щербина:

$$
A_{m}^{\circ}\left(u_{1}, \ldots, u_{m} ; p_{1}, p_{2}, \ldots\right)=\sum_{\mu}\left(\prod_{w \in \mu} \prod_{i=1}^{m}\left(1+u_{i} c(w)\right)\right) \frac{\operatorname{dim}_{\mu}}{|\mu| !} s_{\mu}\left(p_{1}, p_{2}, \ldots\right) .
$$

Действительно, обозначим через $C^{(k)} \in Z \mathbb{C} S_{n}$ сумму всех перестановок вырожденности $k$. Тогда $a_{k_{1}, \ldots, k_{m} ; \mu}^{\circ}$ равно коэффициенту при $C_{\mu} /\left|C_{\mu}\right|$ в разложении элемента $(1 / n !) C^{\left(k_{1}\right)} \cdots C^{\left(k_{m}\right)}$ по базису $C_{\mu}$. Поэтому вычисление производящей функции для этих чисел сводится при помощи предложения 2.4 к вычислению собственных чисел оператора умножения на $C^{(k)}$ в $Z \mathbb{C} S_{n}$. Для вычисления этих собственных чисел заметим, что $C^{(k)}$ выражается как $k$-я элементарная симметрическая функция от элементов Юциса-Мерфи:

$$
C^{(k)}=\sigma_{k}\left(X_{1}, \ldots, X_{n}\right), \quad \sum_{k=0}^{n} C^{(k)} u^{k}=\prod_{i=1}^{n}\left(1+X_{i} u\right) .
$$

Это следует из того, что всякая перестановка $\tau$ однозначно представляется в виде произведения транспозиций $\tau=\left(a_{1}, b_{1}\right) \cdots\left(a_{k}, b_{k}\right), a_{i}<b_{i}$, которое удовлетворяет следующему условию строгой монотонности для участвующих в нем транспозиций: $b_{1}<\cdots<b_{k}$. (Для доказательства достаточно заметить, что в качестве $b_{k}$ может выступать только наибольший элемент, не оставляемый на месте перестановкой $\tau$, после чего можно рассуждать по индукции.) Более того, количество $k$ транспозиций в таком произведении в точности равно вырожденности перестановки $\tau$. Применяя формулу (6) предложения 2.5, мы получаем формулу (9) для производящей функции для обобщенных чисел Гурвица.

ЗАмЕчАниЕ 2.8. Приведенную формулу для собственных чисел элемента $\sum_{k=0}^{n} C^{(k)} u^{k} \in Z \mathbb{C} S_{n}$ можно записать также в виде тождества для специализации функций Шура, представленного в следующих двух эквивалентных формах:

$$
\begin{gathered}
s_{\mu}(v, v, v, \ldots)=\frac{\operatorname{dim}_{\mu}}{|\mu| !} \prod_{w \in \mu}(v+c(w)), \\
\left.s_{\mu}\left(p_{1}, p_{2}, \ldots\right)\right|_{p_{i}=u^{-1}}=u^{|\mu|} s_{\mu}\left(u^{-1}, u^{-1}, \ldots\right)=\frac{\operatorname{dim}_{\mu}}{|\mu| !} \prod_{w \in \mu}(1+u c(w)) .
\end{gathered}
$$

Пример 2.9 (числа Буске-Мелу-Шеффера). Числа Буске-Мелу-Шеффера, как и обобщенные числа Гурвица, перечисляют разложения перестановки данного циклового типа в произведение данного числа перестановок произвольных цикловых типов. При этом, в отличие от обобщенных чисел Гурвица, фиксируется не вырожденность каждой из перестановок, участвующих в произведении, а их суммарная вырожденность. Эти числа были введены в [5]. 
Положим

$$
b_{m, k ; \mu}^{\circ}=\frac{1}{|\mu| ! \mid}\left|\left\{\left(\tau_{1}, \ldots, \tau_{m}\right), \tau_{i} \in S_{|\mu|}: \sum_{i=1}^{m} k\left(\tau_{i}\right)=k, \tau_{1} \circ \cdots \circ \tau_{m} \in C_{\mu}\left(S_{|\mu|}\right)\right\}\right|
$$

и определим числа $b_{m, k ; \mu}$ аналогичным образом, потребовав дополнительно, чтобы подгруппа в $S_{|\mu|}$, порожденная перестановками $\tau_{1}, \ldots, \tau_{\mu}$, действовала на множестве $\{1, \ldots,|\mu|\}$ транзитивно. Производящие функции

$$
\begin{aligned}
& B_{m}^{\circ}\left(u ; p_{1}, p_{2}, \ldots\right)=\sum_{k, \mu} b_{m, k ; \mu}^{\circ} u^{k} p_{\mu_{1}} p_{\mu_{2}} \cdots \\
& B_{m}\left(u ; p_{1}, p_{2}, \ldots\right)=\sum_{k, \mu \neq \varnothing} b_{m, k ; \mu} u^{k} p_{\mu_{1}} p_{\mu_{2}} \cdots
\end{aligned}
$$

связаны между собой соотношением

$$
B_{m}^{\circ}=\exp \left\{B_{m}\right\} .
$$

Они получаются, очевидно, из производящих функций для обобщенных чисел Гурвица, если положить $u_{1}=\cdots=u_{m}=u$ :

$$
\begin{aligned}
& B_{m}^{\circ}\left(u ; p_{1}, p_{2}, \ldots\right)=A_{m}^{\circ}\left(u, \ldots, u ; p_{1}, p_{2}, \ldots\right), \\
& B_{m}\left(u ; p_{1}, p_{2}, \ldots\right)=A_{m}\left(u, \ldots, u ; p_{1}, p_{2}, \ldots\right) .
\end{aligned}
$$

Отсюда вытекает, что справедливо следующее утверждение.

Теорема 2.10 [8]. Производящая функиия $B_{m}^{\circ}\left(u ; p_{1}, p_{2}, \ldots\right)$ для чисел Буске-Мелу-Шефбера является однопараметрическим семейством т-функиии для иерархии KП, а ее логарифм $B_{m}\left(u ; p_{1}, p_{2}, \ldots\right)$ является однопараметрическим семейством решений этой иерархии.

Соответственно, производящая функция $B_{m}^{\circ}$ соответствует набору значений параметров

$$
y_{c}=(1+u c)^{m} \quad \text { для } \quad c=\ldots,-2,-1,0,1,2, \ldots
$$

в семействе Орлова-Щербина:

$$
B_{m}^{\circ}\left(u ; p_{1}, p_{2}, \ldots\right)=\sum_{\mu} \prod_{w \in \mu}(1+u c(w))^{m} \frac{\operatorname{dim}_{\mu}}{|\mu| !} s_{\mu}\left(p_{1}, p_{2}, \ldots\right) .
$$

ЗАмЕчАниЕ 2.11. Если в определении чисел Буске-Мелу-Шеффера не накладывать ограничения на вырожденность перестановок, т. е. положить $u=1$ в производящей функции для этих чисел, то ответ становится совсем простым:

$$
B_{m}^{\circ}\left(1 ; p_{1}, p_{2}, \ldots\right)=\sum_{k=0}^{\infty}(k !)^{m-1} s_{k}(p) .
$$

Действительно, при $u=1$ произведение $\prod_{w \in \mu}(1+u c(w))$ отлично от нуля, только если диаграмма Юнга $\mu$ состоит из одной строки, и равно в этом случае $|\mu| !$. 
ПримеР 2.12 (монотонные простые числа Гурвица). Монотонные простые числа Гурвица определяются следующим образом [7]. Обозначим через $\vec{h}_{m ; \mu}^{\circ}$ (соответственно $\vec{h}_{m ; \mu}$ ) результат деления на $|\mu|$ ! количества последовательностей из $m$ транспозиций $\tau_{1}, \ldots, \tau_{m}$ в $S_{|\mu|}$, обладающих следующими свойствами:

- перестановка $\tau_{1} \circ \cdots \circ \tau_{m}$ имеет цикловой тип $\mu$ (соответственно, подгруппа, порожденная этими транспозициями, действует на множестве $\{1, \ldots, n\}$ транзитивно);

- если записать каждую транспозицию $\tau_{i}$ парой переставляемых ею элементов $\left(a_{i}, b_{i}\right)$, так что $a_{i}<b_{i}$, то $b_{1} \leqslant b_{2} \leqslant \cdots \leqslant b_{m}$ (условие монотонности).

Как и в случае обычных чисел Гурвица, введем две производящие функции

$$
\begin{aligned}
\vec{H}^{\circ}\left(u ; p_{1}, p_{2}, \ldots\right) & =\sum_{m=0}^{\infty} \sum_{\mu} \vec{h}_{m ; \mu}^{\circ} p_{\mu_{1}} p_{\mu_{2}} \cdots u^{m} \\
\vec{H}\left(u ; p_{1}, p_{2}, \ldots\right) & =\sum_{m=1}^{\infty} \sum_{\mu \neq \varnothing} \vec{h}_{m ; \mu} p_{\mu_{1}} p_{\mu_{2}} \cdots u^{m} .
\end{aligned}
$$

Тогда справедливо следующее утверждение.

Теорема 2.13 [7], [12]. Производящая функиия $\vec{H}^{\circ}\left(u ; p_{1}, p_{2}, \ldots\right)$ для простых монотонных чисел Гурвица является однопараметрическим семейством $\tau$-функиий для иерархии КП, а ее логарифм $\vec{H}\left(u ; p_{1}, p_{2}, \ldots\right)$ является однопараметрическим семейством решений этой иерархии.

Соответствующие этому решению параметры $y_{c}$ семейства имеют следующие значения:

$$
y_{c}=\frac{1}{1-u c}, \quad c=\ldots,-2,-1,0,1,2, \ldots
$$

Действительно, рассмотрим сумму всех возможных монотонных разложений длины $m$ в группе $S_{n}$ :

$$
\vec{C}^{(m)}=\sum_{\substack{\left(i_{1}, \ldots, i_{m}\right),\left(j_{1}, \ldots, j_{m}\right) \\ i_{1}<j_{1}, \ldots, i_{m}<j_{m} \\ j_{1} \leqslant \cdots \leqslant j_{m}}}\left(i_{1}, j_{1}\right) \cdots\left(i_{m}, j_{m}\right)
$$

С одной стороны, монотонные числа Гурвица - это коэффициенты в разложении элемента $(1 / n !) \vec{C}^{(m)}$ по базису $C_{\mu} /\left|C_{\mu}\right|$, и, следовательно, производящая функция для этих чисел определяется собственными числами оператора умножения на $\vec{C}(m)$. С другой стороны, легко видеть, что $\vec{C}^{(m)}$ выражается как $m$-я полная симметрическая функция от элементов Юциса-Мерфи:

$$
\vec{C}^{(m)}=h_{m}\left(X_{1}, \ldots, X_{n}\right), \quad \sum_{m=0}^{\infty} \vec{C}^{(m)} u^{m}=\prod_{k=1}^{n} \frac{1}{1-u X_{k}}
$$

откуда и вытекает указанная выше специализация для производящей функции монотонных чисел Гурвица. 
Интересно отметить, что из приведенной специализации значений параметров семейства Орлова-Щербина вытекает, что монотонные числа Гурвица, с точностью до знака, соответствуют формальному значению параметра $m=-1$ для чисел Буске-Мелу-Шеффера:

$$
\vec{h}_{m, \mu}=(-1)^{m} b_{-1, m ; \mu}, \quad \vec{H}\left(u, p_{1}, p_{2}, \ldots\right)=B_{-1}\left(-u, p_{1}, p_{2}, \ldots\right) .
$$

ЗАмечАниЕ 2.14 (алгебра Керова-Ольшанского). В приведенном анализе формулы характеров число элементов $n$ в группе перестановок $S_{n}$ считается фиксированным, несмотря на то, что в производящие ряды собираются числа, соответствующие различным значениям этого параметра. С. В. Керов и Г.И. Ольшанский [18] предложили естественный способ объединить алгебры $Z \mathbb{C} S_{n}$ для различных $n$ в одну большую алгебру, установив тем самым регулярную зависимость от $n$ рассматриваемых комбинаторных чисел.

Рассмотрим счетное множество элементов, скажем, $\mathbb{N}$. Рассмотрим множество всевозможных пар следующего вида: (конечное подмножество в $\mathbb{N}$, перестановка этого подмножества). На множестве таких пар имеется естественная полугрупповая операция: подмножества, соответствующие двум таким парам, объединяются, а перестановки перемножаются. Алгеброй Керова-Ольшанского $\mathscr{W}$ называется центр (полу)групповой алгебры этой полугруппы. Для всякого разбиения $\mu$ обозначим через $C_{\mu}$ элемент алгебры, равный сумме всех возможных пар следующего вида: $(|\mu|$-элементное подмножество в $\mathbb{N}$, перестановка этого подмножества циклового типа $\mu$ ). Тогда элементы $C_{\mu}$ для всевозможных разбиений $\mu$ образуют аддитивный базис в алгебре $\mathscr{W}$. Вот несколько примеров умножения в этой (коммутативной и ассоциативной) алгебре:

$$
\begin{aligned}
C_{1^{1}}^{2} & =C_{1^{1}}+2 C_{1^{2}}, \\
C_{1^{1}} C_{2^{1}} & =2 C_{2^{1}}+C_{1^{1} 2^{1}}, \\
C_{2^{1}}^{2} & =3 C_{3^{1}}+C_{1^{2}}+2 C_{2^{2}}, \\
C_{2^{1}} C_{1^{1} 2^{1}} & =3 C_{3^{1}}+4 C_{2^{2}}+3 C_{1^{1} 3^{1}} 3 C_{1^{3}}+2 C_{1^{1} 2^{2}} .
\end{aligned}
$$

Для каждого натурального $n$ имеется естественный гомоморфизм $\phi_{n}: \mathscr{W} \rightarrow$ $Z \mathbb{C} S_{n}$, задаваемый “забыванием носителя перестановки”:

$$
\phi_{n}\left(C_{\mu}\right)=\left(\begin{array}{c}
n-|\mu|+\varepsilon(\mu) \\
\varepsilon(\mu)
\end{array}\right) C_{1^{n-|\mu|} \mu},
$$

где $\varepsilon(\mu)$ - число единиц в разбиении $\mu$. В частности,

$$
\phi_{n}\left(C_{\mu}\right)= \begin{cases}C_{\mu}, & \text { если }|\mu|=n, \\ 0, & \text { если }|\mu|>n .\end{cases}
$$

Наличие таких гомоморфизмов позволяет поставить в соответствие всякому элементу $a \in \mathscr{W}$ функцию на множестве диаграмм Юнга: $\lambda \mapsto f_{a}(\lambda)$, где $f_{a}(\lambda)$ - собственное число элемента $\phi_{|\lambda|}(a) \in Z \mathbb{C} S_{|\lambda|}$, отвечающее разбиению $\lambda$. Теорема Керова-Ольшанского (в современной интерпретации) утверждает, что для всякого $a \in \mathscr{W}$ функция $f_{a}$ на множестве диаграмм Юнга является симметрическим многочленом от содержаний диаграмм, более того, это соответствие устанавливает изоморфизм алгебры $\mathscr{W}$ с алгеброй симметрических функций. В другом варианте теорема Керова-Ольшанского интерпретирует $f_{a}(\lambda)$ 
как значение некоторого симметрического многочлена от сдвинутых частей $\lambda_{1}-1, \lambda_{2}-2, \lambda_{3}-3, \ldots$ разбиения $\lambda=\left(\lambda_{1}, \lambda_{2}, \ldots\right), \lambda_{1} \geqslant \lambda_{2} \geqslant \cdots$. Поэтому алгебру $\mathscr{W}$ часто называют также алгеброй сдвинутых симметрических функиий (см. [24]).

В контексте чисел Гурвица предложение 2.5 утверждает, что для всякого симметрического многочлена (от неопределенного числа образующих) значение этого многочлена на элементах Юциса-Мерфи корректно задает элемент алгебры Керова-Ольшанского. Отсюда и вытекает регулярная зависимость рассматриваемых чисел от мощности $n=|\mu|$ рассматриваемых разбиений.

2.3. Разложение по родам. Числа Гурвица имеют непосредственное отношение к перечислению накрытий проективной прямой. А именно, числа Гурвица $h_{m, \mu}^{\circ}$ перечисляют разветвленные накрытия проективной прямой $\mathbb{C} P^{1}$ с фиксированными точками ветвления в ней такими, что ветвление над одной из них имеет заданный цикловой тип $\mu$, а над оставшимися $m$ точками является простым. Разветвленные накрытия перечисляются с весом: вес накрытия обратно пропорционален порядку группы его автоморфизмов (последняя, как правило, тривиальна). Род накрывающей поверхности определяется данными ветвления однозначно, по формуле Римана-Гурвица, однако эта поверхность может оказаться несвязной, поэтому лучше говорить о ее эйлеровой характеристике. В свою очередь, логарифм $H\left(u ; p_{1}, p_{2}, \ldots\right) \tau$-функции $H^{\circ}$ перечисляет связные разветвленные накрытия. В случае связного разветвленного накрытия проективной прямой с ветвлением циклового типа $\mu$ над одной точкой и простыми ветвлениями над $m$ другими точками формула Римана-Гурвица для рода g накрывающей поверхности принимает вид

$$
2 g-2=m-|\mu|-\ell(\mu) .
$$

Она подсказывает, как явно выделить род $g$ связной накрывающей поверхности в производящей функции: всякая дополнительная точка простого ветвления должна добавлять единицу в степень параметра, отвечающего за этот род, а часть длины $i$ в разбиении $\mu$ должна уменьшать эту степень на $i+1$. Как результат, мы должны сделать замену переменных $p_{i} \mapsto \hbar^{-i-1} p_{i}$, что приводит к деформации функций Шура:

$$
s_{\lambda}^{\hbar}\left(p_{1}, p_{2}, \ldots\right)=s_{\lambda}\left(\frac{p_{1}}{\hbar^{2}}, \frac{p_{2}}{\hbar^{3}}, \frac{p_{3}}{\hbar^{4}}, \ldots\right)
$$

и положить $y_{c}=e^{u \hbar c}$ в параметрах семейства Орлова-Щербина. Тогда производящая функция для связных чисел Гурвица приобретет вид разложения по родам:

$$
\begin{gathered}
H^{\hbar}\left(u ; p_{1}, p_{2}, \ldots\right)=\hbar^{2} \log \left(\sum_{\mu} e^{u \hbar c(\mu)} \frac{\operatorname{dim}_{\mu}}{|\mu| !} s_{\mu}^{\hbar}\left(p_{1}, p_{2}, \ldots\right)\right) \\
=H_{0}\left(u ; p_{1}, p_{2}, \ldots\right)+\hbar^{2} H_{1}\left(u ; p_{1}, p_{2}, \ldots\right) \\
+\hbar^{4} H_{2}\left(u ; p_{1}, p_{2}, \ldots\right)+\cdots
\end{gathered}
$$


где функция $H_{g}, g=0,1,2, \ldots$, перечисляет разветвленные накрытия рода $g$. В свою очередь, иерархия уравнений КП также продеформируется. В частности, первое уравнение КП (1) на продеформированную функцию приобретает вид

$$
\frac{\partial^{2} H^{\hbar}}{\partial p_{2}^{2}}=\frac{\partial^{2} H^{\hbar}}{\partial p_{1} \partial p_{3}}-\frac{1}{2}\left(\frac{\partial^{2} H^{\hbar}}{\partial p_{1}^{2}}\right)^{2}-\frac{\hbar^{2}}{12} \frac{\partial^{4} H^{\hbar}}{\partial p_{1}^{4}}
$$

(мы пользуемся равенством $\left.H^{\hbar}\left(u ; p_{1}, p_{2}, \ldots\right)=\hbar^{2} H\left(\hbar u ; p_{1} / \hbar^{2}, p_{2} / \hbar^{3}, \ldots\right)\right)$. Это означает, например, что производящая функция $H_{0}$ для связных чисел Гурвица рода 0 является решением бездисперсионного уравнения КП:

$$
\frac{\partial^{2} H_{0}}{\partial p_{2}^{2}}=\frac{\partial^{2} H_{0}}{\partial p_{1} \partial p_{3}}-\frac{1}{2}\left(\frac{\partial^{2} H_{0}}{\partial p_{1}^{2}}\right)^{2} .
$$

Следующий член разложения по степеням параметра $\hbar$ удовлетворяет уравнению

$$
\frac{\partial^{2} H_{1}}{\partial p_{2}^{2}}=\frac{\partial^{2} H_{1}}{\partial p_{1} \partial p_{3}}-\frac{\partial^{2} H_{0}}{\partial p_{1}^{2}} \frac{\partial^{2} H_{1}}{\partial p_{1}^{2}}-\frac{1}{12} \frac{\partial^{4} H_{0}}{\partial p_{1}^{4}},
$$

которое, в отличие от исходного уравнения КП, линейно относительно неизвестной функции $H_{1}$. Поэтому, зная член разложения нулевого рода $H_{0}$, следующий член разложения $H_{1}$ можно найти стандартными методами теории линейных дифференциальных уравнений. То же самое справедливо и для всех старших родов.

Для случая чисел Гурвица член нулевого рода $H_{0}$ известен со времен Гурвица. Его коэффициенты имеют вид

$$
h_{m ; \mu}=|\mu|^{\ell(\mu)-3} \frac{m !}{|\operatorname{Aut}(\mu)|} \prod_{i=1}^{\ell(\mu)} \frac{\mu_{i}^{\mu_{i}}}{\mu_{i} !}, \quad m=|\mu|+\ell(\mu)-2 .
$$

Аналогично случаю обычных чисел Гурвица, все остальные их вариации также интерпретируются как числа разветвленных накрытий сферы с заданными ограничениями на типы ветвления в заданных точках. При этом условие транзитивности действия группы, порожденной перестановками, участвующими в произведении, эквивалентно условию связности поверхности накрытия. Род этой поверхности определяется данными ветвления по формуле Римана-Гурвица и может быть выделен явно подходящей перенормировкой параметров. Более того, мы можем ввести понятие разложения по родам для произвольных решений иерархии КП, принадлежащих семейству Орлова-Щербина.

Пусть

$$
\varphi(c)=d_{0}+d_{1} c+d_{2} c^{2}+d_{3} c^{3}+\cdots
$$

- произвольный степенной ряд.

ОПРЕДЕЛЕНИЕ 5. Разложением по родам $\tau$-функции иерархии КП, получаемой из функции семейства Орлова-Щербина

$$
\sum_{\mu} y_{\mu} \frac{\operatorname{dim}_{\mu}}{|\mu| !} s_{\mu}\left(p_{1}, p_{2}, \cdots\right)
$$


подстановкой $y_{c}=\varphi(c), c=\ldots,-2,-1,0,1,2$, называется функция

$$
\sum_{\mu}\left(\prod_{w \in \mu} \varphi(\hbar c(w))\right) \frac{\operatorname{dim}_{\mu}}{|\mu| !} s_{\mu}^{\hbar}\left(p_{1}, p_{2}, \ldots\right) .
$$

Докажем, что это определение корректно.

ТЕОРема 2.15. Для любого степенного ряда (15) разложение по степеням параметра ћ функиии

$$
\Phi^{\hbar}\left(p_{1}, p_{2}, \ldots\right)=\hbar^{2} \log \sum_{\mu}\left(\prod_{w \in \mu} \varphi(\hbar c(w))\right) \frac{\operatorname{dim}_{\mu}}{|\mu| !} s_{\mu}^{\hbar}\left(p_{1}, p_{2}, \ldots\right)
$$

содержит только четные неотрицательные степени параметра $\hbar$.

Тем самым, действительно имеет смысл говорить о роде каждого монома в степенном разложении.

По-видимому, сформулированная теорема допускает непосредственное доказательство, не привязанное к разветвленным накрытиям сферы. Мы, однако, воспользуемся результатом Ги-Паке и Харнада [11], который интерпретирует функцию $\Phi^{\hbar}$ как производящую функцию для количества разветвленных накрытий сферы связными поверхностями, подсчитанных с определенными весами. Рассмотрим (нестрого) монотонное разложение перестановки из $n$ элементов в произведение транспозиций

$$
\left(i_{1}, j_{1}\right)\left(i_{2}, j_{2}\right) \cdots\left(i_{m}, j_{m}\right), \quad i_{k}<j_{k}, \quad j_{1} \leqslant j_{2} \leqslant \cdots \leqslant j_{m} .
$$

Назовем весом такого разложения моном $d_{k_{1}} \cdots d_{k_{n}}$ от формальных переменных $d_{0}, d_{1}, \ldots$, в котором $k_{s}$ - количество транспозиций в разложении, у которых второй (больший) индекс равен $s$. Соответственно, общее число транспозиций в разложении равно $m=k_{1}+\cdots+k_{n}$. Тогда $\tau$-функция определения 5 служит производящей функцией для чисел нестрого монотонных разложений, посчитанных с учетом весов, а функция $\Phi^{\hbar}$ теоремы - производящей функцией для аналогичных разложений, удовлетворяющих условию транзитивности. Соответственно, степень переменной $\hbar$ в каждом мономе функции $\Phi^{\hbar}$ равна удвоенному роду поверхности накрытия, задаваемого разложением. Действительно, рассмотрим произведение

$$
\varphi\left(X_{1}\right) \varphi\left(X_{2}\right) \cdots \varphi\left(X_{n}\right)
$$

(как элемент кольца $\left.Z \mathbb{C} S_{n}\left[\left[d_{0}, d_{1}, \ldots\right]\right]\right)$, где $X_{1}, \ldots, X_{n}$ - элементы ЮцисаМерфи. Тогда, как нетрудно видеть, это произведение равно сумме всех возможных нестрого монотонных разложений в произведение транспозиций в группе $S_{n}$ (для фиксированного $n$ ), взятых с коэффициентами, равными весам соответствующих разложений. Из этого наблюдения и вытекает теорема Ги-Паке-Харнада, аналогично доказательствам теорем 2.7, 2.10 и 2.13.

ПримеР 2.16. Производящая функция $B_{m}$ для связных чисел Буске-МелуШеффера отвечает набору параметров $y_{c}=(1+u c)^{m}$ в семействе Орлова-Щербина. Для получения ее разложения по родам $B_{m}^{\hbar}$ нужно заменить эти параметры на $y_{c}=(1+u \hbar c)^{m}$ :

$$
B_{m}^{\hbar}=\hbar^{2} \log \sum_{\mu}\left(\prod_{w \in \mu}(1+u \hbar c(w))^{m}\right) \frac{\operatorname{dim}_{\mu}}{|\mu| !} s_{\mu}^{\hbar}\left(p_{1}, p_{2}, \ldots\right) .
$$


Коэффициенты этой функции перечисляют связные накрытия проективной прямой поверхностью заданного рода с фиксированными точками ветвления, над одной из которых ветвление имеет заданный цикловой тип $\mu$, а все остальные точки ветвления находятся среди оставшихся $m$ точек и типы ветвления над ними могут быть произвольными. Для количества накрытий нулевого рода в [5] была получена элегантная явная формула:

$$
b_{m, k, \mu}=\frac{m(m|\mu|-k+1)^{\overline{\ell(\mu)-3}}}{|\operatorname{Aut}(\mu)|} \prod_{i=1}^{\ell(\mu)}\left(\begin{array}{c}
m \mu_{i}-1 \\
\mu_{i}
\end{array}\right), \quad k=|\mu|+\ell(\mu)-2,
$$

где через

$$
(d+1)^{\bar{r}}=(d+1)(d+2) \cdots(d+r)
$$

обозначено восходящее произведение $r$ сомножителей, и для $r<0$ мы полагаем

$$
(d+1)^{\bar{r}}=\frac{1}{(d+r+1)^{\overline{-r}}} .
$$

ПримеР 2.17. Для монотонных чисел Гурвица разложение по родам имеет вид

$$
\vec{H}^{\hbar}=\hbar^{2} \log \sum_{\mu} \prod_{w \in \mu} \frac{1}{1-u \hbar c(w)} \frac{\operatorname{dim}_{\mu}}{|\mu| !} s_{\mu}^{\hbar}\left(p_{1}, p_{2}, \ldots\right) .
$$

Формула для монотонных чисел Гурвица рода 0 получена в [6]. С точностью до знака $(-1)^{m}$ она получается формальной подстановкой $m=-1, k=m$ в приведенную выше формулу для чисел Буске-Мелу-Шеффера рода 0:

$$
\vec{h}_{m, \mu}=\frac{(2|\mu|+1)^{\overline{\ell(\mu)-3}}}{|\operatorname{Aut}(\mu)|} \prod_{i=1}^{\ell(\mu)}\left(\begin{array}{c}
2 \mu_{i} \\
\mu_{i}
\end{array}\right), \quad m=|\mu|+\ell(\mu)-2 .
$$

2.4. Иерархия решетки Тоды. Теорема 2.1 является специализацией следующего более общего результата. Рассмотрим два набора независимых переменных $p=\left(p_{1}, p_{2}, \ldots\right)$ и $q=\left(q_{1}, q_{2}, \ldots\right)$ и еще дополнительный набор формальных параметров $\left(\ldots, y_{-1}, y_{0}, y_{1}, y_{2}, \ldots\right)$. Для всякого $n \in \mathbb{Z}$ положим

$$
\tau_{n}\left(p_{1}, p_{2}, \ldots ; q_{1}, q_{2}, \ldots\right)=r_{0}(n) \sum_{\mu} r_{\mu}(n) s_{\mu}\left(p_{1}, p_{2}, \ldots\right) s_{\mu}\left(q_{1}, q_{2}, \ldots\right)
$$

где суммирование идет по всем разбиениям $\mu$ и

$$
r_{\mu}(n)=\prod_{w \in \mu} y_{c(w)+n}, \quad r_{0}(n)= \begin{cases}\prod_{j=1}^{n-1} y_{j}^{n-j}, & n \geqslant 0, \\ \prod_{j=n+1}^{0} y_{j}^{j-n}, & n<0 .\end{cases}
$$

ТЕОРема 2.18. Каждая из функиий $\tau_{n}$ является $\tau$-функцией иерархии КП как относительно р-переменных, так и относительно q-переменных. 
На самом деле справедливо более сильное утверждение: совокупность функций $\tau_{n}, n \in \mathbb{Z}$, образует набор $\tau$-функций иерархии решетки Тоды. Иерархия решетки Тоды [29] содержит уравнения иерархии КП на $\log \tau_{n}$ по каждой из двух групп переменных и для каждого $n$, а также дополнительный набор уравнений, в которые включены производные по переменным из обеих групп от функций с тремя соседними номерами. Простейшее из таких уравнений имеет вид

$$
\frac{\partial^{2} \log \tau_{n}}{\partial p_{1} \partial q_{1}}=\frac{\tau_{n-1} \tau_{n+1}}{\tau_{n}^{2}}, \quad n \in \mathbb{Z} .
$$

Как и в случае иерархии КП, геометрически более наглядным является описание не самих уравнений иерархии решетки Тоды, а пространства их решений. Решения этой иерархии параметризуются бесконечномерными матрицами - элементами $M$ группы $\mathrm{GL}(V)$ (см. [15; гл. 4]). Для простоты мы ограничимся случаем, когда матрица $M$ верхнетреугольная

$$
M: z^{k} \mapsto \sum_{i \geqslant k} M_{i, k} z^{i}, \quad M_{k, k} \neq 0
$$

При $M=\mathrm{Id}$ соответствующее решение имеет вид

$$
\tau_{n}=\exp \left\{\sum_{k} \frac{p_{k} q_{k}}{k}\right\}=\sum_{\mu} s_{\mu}(p) s_{\mu}(q)
$$

для всех $n$. При бозон-фермионном соответствии (относительно $p$-переменных) такой функции $\tau_{n}$ сопоставляется разложимое внешнее произведение, имеющее вид $\beta_{1} \wedge \beta_{2} \wedge \cdots$, в котором

$$
\beta_{k}=\exp \left\{\sum_{i} \frac{q_{i} z^{i}}{i}\right\} z^{n-k}=\sum_{j=0}^{\infty} s_{j}(q) z^{n-k+j} .
$$

При $n \neq 0$ такое внешнее произведение не лежит в $\Lambda^{\infty / 2} V$; оно лежит в другом пространстве, обозначаемом через $\Lambda^{\infty / 2+n} V$ и называемом пространством фермионов заряда $n$. Как и в случае заряда 0 , базисные векторы пространства $\Lambda^{\infty / 2+n} V$ нумеруются разбиениями. Они имеют вид

$$
v_{\mu}=z^{m_{1}} \wedge z^{m_{2}} \wedge z^{m_{3}} \wedge \cdots, \quad m_{i}=\mu_{i}-i+n .
$$

Для общего элемента $M \in \mathrm{GL}(V)$ фермион, сопоставляемый $\tau$-функции $\tau_{n}$, модифицируется следующим образом:

$$
\tau_{n} \leftrightarrow r_{0}(n) \frac{M\left(\beta_{1}\right)}{M_{n-1, n-1}} \wedge \frac{M\left(\beta_{2}\right)}{M_{n-2, n-2}} \wedge \cdots,
$$

где

$$
r_{0}(n)= \begin{cases}\prod_{j=0}^{n-1} M_{j, j}, & n \geqslant 0 \\ \prod_{j=n}^{-1} M_{j, j}^{-1}, & n<0\end{cases}
$$


Нормировочный множитель $r_{0}(n)$ в правой части (16) обязан своим происхождением бесконечномерности рассматриваемых пространств. Этот множитель не зависит от $n$ и равен $\prod_{i=1}^{\infty} M_{-i,-i}$, но только его части перегруппированы таким образом, чтобы коэффициент при члене младшей степени $k$-го сомножителя внешнего произведения (т. е. при $z^{n-k}$ ) был равен 1 для достаточно больших $k$. Легко видеть, что решение иерархии, фигурирующее в теореме 2.18 , соответствует случаю, когда $M$ - диагональная матрица из замечания 2.2.

ПримеР 2.19 (двойные числа Гурвица и иерархия решетки Тоды). Исторически именно утверждение о том, что производящая функция для двойных чисел Гурвица является $\tau$-функцией интегрируемой иерархии, доказанное А. Окуньковым в работе [23], послужило прототипом утверждений подобного рода.

Для определения двойных чисел Гурвица рассмотрим для данного натурального числа $m$ всевозможные наборы перестановок $\left(\alpha, \beta, \tau_{1}, \ldots, \tau_{m}\right)$, в которых

- перестановка $\alpha$ принадлежит цикловому типу, заданному фиксированным разбиением $\mu \vdash|\mu|$, и лежит, тем самым, в группе $S_{|\mu|}$;

- перестановка $\beta \in S_{|\mu|}$ принадлежит цикловому типу, заданному фиксированным разбиением $\nu \vdash|\nu|=|\mu|$;

- перестановки $\tau_{1}, \ldots, \tau_{m}$ являются транспозициями;

- перестановка $\alpha \circ \beta \circ \tau_{1} \circ \cdots \circ \tau_{m}$ является тождественной.

Двойным числом Гурвища $d_{m ; \mu, \nu}^{\circ}$ (соответственно связным двойным числом Гурвища $\left.d_{m ; \mu, \nu}\right)$ называется величина

$$
\begin{gathered}
d_{m ; \mu, \nu}^{\circ}=\frac{1}{|\mu| !} \mid\left\{\left(\alpha, \beta, \tau_{1}, \ldots, \tau_{m}\right): \alpha \in C_{\mu}\left(S_{|\mu|}\right), \beta \in C_{\nu}\left(S_{|\mu|}\right),\right. \\
\left.\tau_{i} \in C_{2}\left(S_{|\mu|}\right), \alpha \circ \beta \circ \tau_{1} \circ \cdots \circ \tau_{m}=\mathrm{id}\right\} \mid
\end{gathered}
$$

(соответственно определение величины $d_{m ; \mu, \nu}$ отличается дополнительным требованием транзитивности действия подгруппы в $S_{|\mu|}$, порождаемой перестановками $\left.\alpha, \beta, \tau_{1}, \ldots, \tau_{m}\right)$.

Двойные числа Гурвица перечисляют разветвленные накрытия двумерной сферы, имеющие ветвления заданных цикловых типов $\mu$ и $\nu$ над двумя выделенными критическими значениями и простые ветвления над всеми остальными критическими значениями. Подходящим выбором комплексной координаты $z$ в накрываемой сфере можно добиться того, чтобы выделенные критические значения имели координаты $z=0$ и $z=\infty$. Связные двойные числа Гурвица перечисляют связные накрытия.

Соберем двойные и связные двойные числа Гурвица в две производящие функции, каждая из которых зависит от двух бесконечных семейств переменных $q_{1}, q_{2}, \ldots$ и $p_{1}, p_{2}, \ldots$ и дополнительных параметров $u, v$ :

$$
\begin{aligned}
D^{\circ}\left(u, v ; p_{1}, p_{2}, \ldots ; q_{1}, q_{2}, \ldots\right) & =\sum_{m=0}^{\infty} \sum_{\mu, \nu} d_{m ; \mu, \nu}^{\circ} p_{\mu_{1}} p_{\mu_{2}} \cdots q_{\nu_{1}} q_{\nu_{2}} \cdots v^{|\mu|} \frac{u^{m}}{m !} \\
D\left(u, v ; p_{1}, p_{2}, \ldots ; q_{1}, q_{2}, \ldots\right) & =\sum_{m=1}^{\infty} \sum_{\mu, \nu} d_{m ; \mu, \nu} p_{\mu_{1}} p_{\mu_{2}} \cdots q_{\nu_{1}} q_{\nu_{2}} \cdots v^{|\mu|} \frac{u^{m}}{m !} .
\end{aligned}
$$


ТеОРема 2.20 [23]. Производящая функиия $D^{\circ}$ является семейством $\tau$-функций иерархии решетки Тодъ.

ПримеР 2.21. Аналогично двойным числам Гурвица определяются обобщенные двойные числа Гурвица, двойные числа Буске-Мелу-Шеффера и двойные монотонные числа Гурвица. Производящие функции для таких вариантов двойных чисел Гурвица зависят от двух наборов времен $\left(p_{1}, p_{2}, \ldots\right)$ и $\left(q_{1}, q_{2}, \ldots\right)$ и дополнительных параметров. Они получаются из функций теоремы 2.18 теми же специализациями параметров $y_{c}$, что были рассмотрены в предыдущем параграфе при рассмотрении соответствующих одинарных чисел. Как следствие, эти производящие функции удовлетворяют уравнениям иерархии КП по каждому набору времен и уравнениям иерархии решетки Тоды, а соответствующие функции для одинарных чисел получаются специализацией $q_{1}=1, q_{i}=0$ $(i>1)$ из производящих функций для двойных чисел.

В самое последнее время круг примеров, разнообразящих наше представление о комбинаторных решениях интегрируемых иерархий, чрезвычайно расширился (см., например, [1]-[3], [10]-[12], [17], [22]). В то же время все известные нам попытки сформулировать и доказать аналогичные свойства интегрируемости для тройных и более общих чисел Гурвица, определение которых требует фиксации полного профиля ветвления над более чем двумя точками сферы, к успеху не привели.

ПримеР 2.22. Ниже нам потребуется следующее семейство решений иерархии решетки Тоды:

$$
\begin{aligned}
N\left(u ; p_{1}, p_{2}, \ldots ; q_{1}, q_{2}, \ldots\right) & =\log \sum_{|\mu|} \prod_{w \in \mu}(u+c(w)) s_{\mu}\left(p_{1}, p_{2}, \ldots\right) s_{\mu}\left(q_{1}, q_{2}, \ldots\right) \\
& =\sum_{|\lambda|=|\mu|} \sum_{k=1}^{|\mu|} n_{k ; \mu, \lambda} u^{k} p_{\lambda} q_{\mu} .
\end{aligned}
$$

Второе равенство служит определением коэффициентов $n_{k ; \lambda, \mu}$. Мы получаем, что $|\lambda| ! n_{k ; \lambda, \mu}($ где $|\lambda|=|\mu|)$ - это количество троек перестановок $\sigma, \alpha, \varphi$ из $|\lambda|$ элементов, удовлетворяющих следующим условиям:

- произведение $\varphi \alpha \sigma$ является тождественной перестановкой;

- перестановки $\alpha$ и $\sigma$ имеют цикловые типы $\mu$ и $\lambda$ соответственно;

- количество циклов в перестановке $\sigma$ равно $k$;

- группа, порожденная перестановками $\varphi, \alpha, \sigma$, действует транзитивно на множестве переставляемых элементов.

Отметим, что, несмотря на очевидную симметрию между перестановками $\varphi$, $\alpha, \sigma$, при построении производящей функции $N$ учитывается полный цикловой тип только у двух из них, а у третьей перестановки учитывается лишь количество циклов.

2.5. Приложения: перечисление карт и триангуляций. Карта на данной поверхности - это граф, вложенный в поверхность и разрезающий ее на двумерные диски. Мы будем предполагать, что поверхность компактна и ориентирована. Две карты считаются эквивалентными, если существует сохраняющий ориентацию гомеоморфизм одной поверхности на другую, переводящий вершины и ребра графа на первой поверхности соответственно в вершины 
и ребра графа на второй поверхности. Карту можно понимать и как результат склейки поверхности из многоугольников - границы многоугольников склеиваются в граф на поверхности.

Для целей настоящей статьи нам будет удобнее использовать следующее комбинаторное определение карты.

ОПРеДЕЛЕНИЕ 6. Пусть $D$ - конечное множество. Картой с множеством полуребер $D$ на ориентированной поверхности называется тройка перестановок $\alpha, \varphi, \sigma$ множества $D$, обладающая следующими свойствами:

- $\alpha$ является инволюцией без неподвижных точек;

- произведение $\varphi \alpha \sigma$ является тождественной перестановкой.

Группа $G=\langle\alpha, \varphi, \sigma\rangle$ перестановок множества $D$, порожденная перестановками $\alpha, \varphi, \sigma$, называется картографической группой карты. Карта называется связной, если ее картографическая группа действует на множестве $D$ транзитивно.

В случае графа, нарисованного на ориентированной поверхности, $D$ представляет собой множество полуребер, или флагов, графа, перестановка $\alpha$ меняет местами концы ребер, перестановка $\varphi$ сдвигает полуребра вдоль каждой грани, а $\sigma$ поворачивает полуребра относительно вершин в положительном направлении (подробнее см. в [34]). Очевидно, что $\alpha$ является инволюцией без неподвижных точек, и легко проверить, что произведение этих трех перестановок действительно является тождественной перестановкой. Карта связна в том и только в том случае, если связна поверхность, на которой она нарисована.

Количество ребер в карте равно половине количества элементов в множестве $D$, т. е. количеству циклов в перестановке $\alpha$. Количество вершин в карте это количество циклов в $\sigma$, степени вершин - это длины этих циклов. Аналогично, количество граней карты равно количеству циклов в перестановке $\varphi$, а степени граней равны длинам этих циклов.

Понятие гиперкарты является обобщением понятия карты. В определении гиперкарты мы освобождаемся от требования, чтобы $\alpha$ была инволюцией без неподвижных точек, восстанавливая таким образом симметрию между тремя перестановками.

Теперь ясно, что перечисление карт и гиперкарт различного вида сводится к перечислению троек перестановок, обладающих различными специальными свойствами, а значит, могут быть применены описанные выше перечислительные методы. В частности, пример 2.22 показывает, что производящая функция, коэффициенты которой перечисляют гиперкарты при заданных наборах валентностей их вершин и граней, является семейством решений иерархии решетки Тоды. Как следствие, она и различные ее специализации, в том числе и перечисляющие карты, являются решениями иерархии КП.

Так, обозначим через $R_{\kappa}^{(n, m)}$ количество связных корневых карт с $n$ ребрами, $m$ гранями и степенями вершин, заданными разбиением $\kappa$ числа $2 n$. Тогда справедлива следующая теорема.

ТЕОРема 2.23 [8]. Производящая функиия

$$
R\left(w, z ; p_{1}, p_{2}, \ldots\right)=\sum_{n, m \geqslant 1} \sum_{\kappa \vdash 2 n} \frac{R_{\kappa}^{(n, m)}}{2 n} p_{\kappa} w^{m} z^{n}
$$


(где для данного разбиения $\kappa=\left(\kappa_{1}, \kappa_{2}, \kappa_{3}, \ldots\right)$ через $p_{\kappa}$ обозначен моном $p_{\kappa}=$ $\left.p_{\kappa_{1}} p_{\kappa_{2}} p_{\kappa_{3}} \cdots\right)$ является двупараметрическим семейством решений уравнений КП.

Действительно, в отличие от числа $R_{\kappa}^{(n, m)}$ корневых карт частное $R_{\kappa}^{(n, m)} /(2 n)$ является взвешенным (т.е. посчитанных с учетом автоморфизмов) числом обычных (некорневых) карт, т. е. равно числу $a_{m, n}(\kappa)$ примера 2.6. Соответственно, функция $R$ теоремы совпадает функцией $A_{m}$ теоремы 2.7 для случая $m=2, u_{1}=w, u_{2}=z$ (альтернативно, функция $R$ является специализацией функции $N$ примера 2.22 при $u=w, q_{i}=z^{i}, i=1,2, \ldots$ ).

При специальных значениях параметров в ряде $R$ мы получаем производящую функцию для корневых кубических карт, т. е. карт, степени всех вершин в которых равны 3. По двойственности это то же самое, что перечисление корневых триангуляций поверхностей произвольного рода. Уравнения КП при этом дают рекуррентные соотношения на количество корневых триангуляций.

Обозначим количество корневых триангуляций поверхности рода $g$ с $2 n$ гранями через $T(n, g)$. Тогда рекуррентные соотношения имеют нижеследующий вид. Введем обозначение

$$
S=\left\{(n, g) \in \mathbb{Z} \times \mathbb{Z}: n \geqslant-1,0 \leqslant g \leqslant \frac{n+1}{2}\right\} .
$$

Теорема 2.24 [8]. Справедливо равенство

$$
T(n, g)=\frac{1}{3 n+2} t(n, g)
$$

где числа $t(n, g)$ определяются квадратичным рекуррентным соотношением

$$
t(n, g)=\frac{4(3 n+2)}{n+1}\left(n(3 n-2) t(n-2, g-1)+\sum t(i, h) t(j, k)\right)
$$

для $(n, g) \in S \backslash\{(-1,0),(0,0)\}$, причем сумма берется по элементам $(i, h) \in S$, $(j, k) \in S$ таким, что $i+j=n-2 u h+k=g$, при начальных условиях

$$
t(-1,0)=\frac{1}{2}, \quad t(n, g)=0 \quad \text { для }(n, g) \notin S .
$$

Это рекуррентное соотношение позволило Э. А. Бендеру, З. Гао и Л. Б. Ричмонду дать чисто комбинаторный вывод постоянного коэффициента в старшем члене асимптотики числа корневых триангуляций при стремлении числа треугольников к бесконечности. Ранее этот коэффициент вычислялся исходя из матричных моделей двумерной квантовой гравитации [31], либо из теории пересечений на пространстве модулей [13].

Теорема 2.25 [4]. Число корневых триангуляиий поверхности рода g, состоящих из 2 п треугольников, имеет асимптотику

$$
T(n, g) \sim \frac{3 b_{g}}{\Gamma(5 g / 2-1 / 2)}\left(\frac{3}{8}\right)^{(g-1) / 2} n^{5(g-1) / 2}(12 \sqrt{3})^{n} \quad \text { nрu } n \rightarrow \infty
$$


при этом константа $b_{g}$ определяется начальным условием $b_{0}=-1$ и квадратичным рекуррентным соотношением

$$
b_{g+1}=\frac{25 g^{2}-1}{24} b_{g}+\frac{1}{2} \sum_{m=1}^{g} b_{g+1-m} b_{m} \quad \text { для } g \geqslant 1 .
$$

Рекуррентное соотношение эквивалентно утверждению о том, что производящая функция для констант $b_{g}$ является решением уравнения Пенлеве-I:

$$
U(y)=\sum_{g=0}^{\infty} \frac{b_{g}}{y^{5(g-1) / 2+2}}, \quad \frac{1}{3} U^{\prime \prime}+U^{2}=y .
$$

Первые несколько значений постоянной $b_{g}$ равны

$$
b_{0}=-1, \quad b_{1}=\frac{1}{24}, \quad b_{2}=\frac{49}{1152}, \quad b_{3}=\frac{1225}{6912}, \quad b_{4}=\frac{4412401}{2654208} .
$$

Эта константа управляет и многими другими аналогичными асимптотиками. В частности, она появляется в [33] при описании модели двумерной квантовой гравитации, в основе которой лежат простые числа Гурвица. А именно, справедливо следующее утверждение.

ТеОРема 2.26. При фиксированном значении рода $g$ имеет место эквивалентность

$$
\frac{h_{2 n+2 g-2 ; 1^{n}}}{(2 n+2 g-2) !} \sim e^{n} n^{5(g-1) / 2-1} \frac{b_{g}}{\Gamma(5 g / 2-1 / 2) 2^{3 g / 2-1 / 2}}
$$

nрu $n \rightarrow \infty$.

Константа $b_{g}$ имеет также явное выражение в терминах чисел пересечений на пространстве модулей стабильных кривых рода $g$ с $n$ отмеченными точками $\overline{\mathscr{M}}_{g ; n}$ (см., например, [13], [19])

$$
b_{g}=\frac{(5 g-5)(5 g-3)}{2^{g}(3 g-3) !} \int_{\overline{\mathscr{M}}_{g ; 3 g-3}} \psi_{1}^{2} \psi_{2}^{2} \cdots \psi_{3 g-3}^{2} .
$$

Отметим имеющий аналогичную описанному выше природу недавний результат П. Г. Зографа, относящийся к перечислению детских рисунков.

Обозначим через $b_{m, n}(\kappa)$ число корневых графов Белого, отвечающих разбиению $\kappa$ с $m$ вершинами и $n$ гранями, и пусть

$$
B_{d}\left(u, v ; p_{1}, p_{2}, \ldots\right)=\sum b_{m, n}(\kappa) u^{m} v^{n} p_{\kappa},
$$

где суммирование ведется по всем $m, n$ и $\kappa \vdash d \ldots$. Положим

$$
\mathscr{B}\left(s, u, v ; p_{1}, p_{2}, \ldots\right)=\sum_{d \geqslant 0} B_{d}\left(u, v ; p_{1}, p_{2}, \ldots\right) s^{d} .
$$

ТЕорема 2.27 [32]. Производящая функиия $\mathscr{B}\left(s, u, v ; p_{1}, p_{2}, \ldots\right)$ является трехпараметрическим семейством решений иерархии КП. 


\section{Список литературы}

[1] A. Alexandrov, A. Mironov, A. Morozov, S. Natanzon, "On KP-integrable Hurwitz functions", J. High Energy Phys., 2014, № 11, 080, 30 pp.; 2014, 21 pp., arXiv: 1405.1395.

[2] N. V. Alexeev, J.E. Andersen, R. C. Penner, P. G. Zograf, Enumeration of chord diagrams on many intervals and their non-orientable analogs, 2014 (v1 - 2013), 23 pp., arXiv: 1307.0967.

[3] J. Ambjørn, L. Chekhov, "The matrix model for dessins d'enfants", Ann. Inst. Henri Poincaré D, 1:3 (2014), 337-361; 2014, 18 pp., arXiv: 1404.4240.

[4] E. A. Bender, Zhicheng Gao, L. B. Richmond, "The map asymptotics constant $t_{g}$ ", Electron. J. Combin., 15:1 (2008), R51, 8 pp.

[5] M. Bousquet-Mélou, G. Schaeffer, "Enumeration of planar constellations", Adv. in Appl. Math., 24:4 (2000), 337-368.

[6] I.P. Goulden, M. Guay-Paquet, J. Novak, "Monotone Hurwitz numbers in genus zero", Canad. J. Math., 65:5 (2013), 1020-1042; 2012, 22 pp., arXiv: 1204.2618.

[7] I. P. Goulden, M. Guay-Paquet, J. Novak, "Polynomiality of monotone Hurwitz numbers in higher genera", Adv. Math., 238 (2013), 1-23.

[8] I. P. Goulden, D. M. Jackson, "The KP hierarchy, branched covers, and triangulations", Adv. Math., 219:3 (2008), 932-951.

[9] Ф. Гриффитс, Дж. Харрис, Принципы алгебраической геометрии, т. 1, 2, Мир, M., 1982, 496 с., 366 с.; пер. с англ.: Ph. Griffiths, J. Harris, Principles of algebraic geometry, Pure and Applied Mathematics, Wiley-Interscience [John Wiley \& Sons], New York, 1978, xii+813 pp.

[10] M. Guay-Paquet, J. Harnad, 2D Toda $\tau$-functions as combinatorial generating functions, 2014, 26 pp., arXiv: 1405.6303.

[11] M. Guay-Paquet, J. Harnad, Generating functions for weighted Hurwitz numbers, 2015 (v1 - 2014), 39 pp., arXiv: 1408.6766.

[12] J. Harnad, A. Yu. Orlov, Hypergeometric $\tau$-functions, Hurwitz numbers and enumeration of paths, 2015 (v1 - 2014), 22 pp., arXiv: 1407.7800.

[13] C. Itzykson, J.-B. Zuber, "Combinatorics of the modular group. II. The Kontsevich integrals", Internat. J. Modern Phys. A, 7:23 (1992), 5661-5705.

[14] A.-A. A. Jucys, "Symmetric polynomials and the center of the symmetric group ring", Rep. Math. Phys., 5:1 (1974), 107-112.

[15] В.Г. Кац, Бесконечномерные алгебры Ли, Мир, М., 1993, 426 с.; пер. с англ.: V. G. Kac, Infinite-dimensional Lie algebras, 3rd ed., Cambridge Univ. Press, Cambridge, 1990, xxii+400 pp.

[16] M. E. Kazarian, S. K. Lando, "An algebro-geometric proof of Witten's conjecture", J. Amer. Math. Soc., 20:4 (2007), 1079-1089.

[17] M. Kazarian, P. Zograf, Virasoro constraints and topological recursion for Grothendieck's dessin counting, 2014, 22 pp., arXiv: 1406.5976.

[18] S. Kerov, G. Olshanski, "Polynomial functions on the set of Young diagrams", C. R. Acad. Sci. Paris Sér. I Math., 319:2 (1994), 121-126.

[19] Yu. I. Manin, P. Zograf, "Invertible cohomological field theories and Weil-Petersson volumes", Ann. Inst. Fourier (Grenoble), 50:2 (2000), 519-535.

[20] Т. Мива, М. Джимбо, Э. Датэ, Солитонъ. Дифберенциальные уравнения, симметрии и бесконечномерные алгебры, МЦНМО, М., 2005, 112 с.; пер. с англ.: T. Miwa, M. Jimbo, E. Date, Solitons. Differential equations, symmetries and infinite dimensional algebras, Cambridge Tracts in Math., 135, Cambridge Univ. Press, Cambridge, 2000, x+108 pp.

[21] G. E. Murphy, "A new construction of Young's seminormal representation of the symmetric groups", J. Algebra, 69:2 (1981), 287-297. 
[22] S. M. Natanzon, A. Yu. Orlov, Hurwitz numbers and BKP hierarchy, 2014, 37 pp., arXiv: 1407.8323 .

[23] A. Okounkov, "Toda equations for Hurwitz numbers", Math. Res. Lett., 7:4 (2000), $447-453$.

[24] А. Окуньков, Г. Ольшанский, "Сдвинутые функции Шура”, Алгебра и анализ, 9:2 (1997), 73-146; англ. пер.: A. Okounkov, G. Olshanskii, "Shifted Schur functions", St. Petersburg Math. J., 9:2 (1998), 239-300.

[25] A. Yu. Orlov, D. M. Scherbin, "Multivariate hypergeometric functions as $\tau$-functions of Toda lattice and Kadomtsev-Petviashvili equation", Phys. D, 152/153 (2001), 51-65.

[26] R. Pandharipande, "The Toda equations and the Gromov-Witten theory of the Riemann sphere", Lett. Math. Phys., 53:1 (2000), 59-74.

[27] B.E. Sagan, The symmetric group. Representations, combinatorial algorithms, and symmetric functions, 2nd ed., Grad. Texts in Math., 203, Springer-Verlag, New York, 2001, xvi+238 pp.

[28] M. Sato, Y. Sato, "Soliton equations as dynamical systems on infinite dimensional Grassmann manifolds", Nonlinear partial differential equations in applied science (Tokyo, 1982), North-Holland Math. Stud., 81, North-Holland, Amsterdam, 1983, 259-271.

[29] K. Ueno, K. Takasaki, "Toda lattice hierarchy", Group representations and systems of differential equations (Tokyo, 1982), Adv. Stud. Pure Math., 4, North-Holland, Amsterdam, 1984, 1-95.

[30] А. М. Вершик, А. Ю. Окуньков, "Новый подход к теории представлений симметрических групп. II", Теория представлений, динамические системы, комбинаторные и алгоритмические методы. Х, Зап. науч. сем. ПОМИ, 307, ПОМИ, СПб., 2004, 57-98; англ. пер.: А. M. Vershik, A. Yu. Okun'kov, "A new approach to representation theory of symmetric groups. II", J. Math. Sci. (N. Y.), 131:2 (2005), 5471-5494.

[31] E. Witten, "Two-dimensional gravity and intersection theory on moduli spaces", Proceedings of the conference on geometry and topology held at Harvard University (Cambridge, MA, 1990), Surveys in differential geometry, 1, International Press, Inc., Boston, MA, 1991, 243-310.

[32] P. Zograf, Enumeration of Grothendieck's dessins and KP hierarchy, 2014 (v1 - 2013), 9 pp., arXiv: 1312.2538.

[33] D. Zvonkine, Enumeration of ramified coverings of the sphere and 2-dimensional gravity, 2005, 26 pp., arXiv: math/0506248v1.

[34] А. К. Звонкин, С. К. Ландо, Графы на поверхностях и их приложения, МЦНМО, M., 2010, 480 с.; пер. с англ.: S. K. Lando, A. K. Zvonkin, Graphs on surfaces and their applications, Encyclopaedia Math. Sci., 141, Low-Dimensional Topology, II, Springer-Verlag, Berlin, 2004, xvi+455 pp.

Максим Эдуардович Казарян

Поступила в редакцию

(Maxim È. Kazarian)

25.12 .2014

Математический институт им. В. А. Стеклова

Российской академии наук;

Национальный исследовательский университет

"Высшая школа экономики"

E-mail: kazarian@mccme.ru

\section{Сергей Константинович Ландо}

(Sergei K. Lando)

Национальный исследовательский университет

"Высшая школа экономики"

E-mail: lando@mccme.ru 